\title{
The DPOSS II compact group survey: first spectroscopically confirmed candidates ${ }^{\star}, \star$
}

\author{
E. Pompei ${ }^{1}$, R. R. de Carvalho ${ }^{2}$, and A. Iovino ${ }^{3}$ \\ 1 European Southern Observatory, Alonso de Cordova 3107, Vitacura, Casilla 19001, Santiago 19, Chile \\ e-mail: epompei@eso.org \\ 2 INPE/MCT, Av. dos Astronautas 1758, S. J. Campos, SP 12227-010, Brazil \\ e-mail: reinaldo@das.inpe.br \\ 3 Osservatorio Astronomico di Brera via Brera 28, 20121 Milano, Italy \\ e-mail: iovino@brera.mi .astro.it
}

Received 11 June 2005 / Accepted 30 August 2005

\section{ABSTRACT}

This paper presents the results of a pilot redshift survey of 18 candidate compact groups from the distant DPOSS survey that extends to redshift $\sim 0.2$ the available surveys of compact groups of galaxies, mainly Hickson Compact Groups and Southern Compact Groups. The goal of our survey was to confirm group membership via redshift information and to measure the characteristic parameters of a representative, albeit small, sample of DPOSS survey groups.

Of the 18 candidates observed, seven are found to be indeed isolated compact groups, i.e. groups with 3 or more concordant members and with no neighbouring known cluster, while 7 are chance projection configurations on the sky. Three remaining candidates, despite having 3 or more concordant member galaxies, are located in the neighbourhood of known clusters, while another candidate turned out to be a dense sub-condensation within Abell 0952.

The median redshift of our 7 confirmed groups is $z \sim 0.12$, to be compared with a median redshift of 0.03 for the local sample of compact groups by Hickson. The typical group size is $\sim 50 \mathrm{kpc}$, and the median radial velocity dispersion is $167 \mathrm{~km} \mathrm{~s}^{-1}$, while typical crossing times range from $0.005 \mathrm{H}_{0}^{-1}$ to $0.03 \mathrm{H}_{0}^{-1}$ with a median value of $0.018 \mathrm{H}_{0}^{-1}$, all similar to the values usually found in the literature for such structures in the local universe. The average mass-to-light ratio for our groups, $M / L_{B}$, is $92 h$, higher than the value found for nearby Hickson compact groups but lower than that found for loose groups. Our results suggest that, once full redshift information for its members becomes available, the DPOSS sample will provide a reference sample to study the properties of compact groups beyond the local universe.

Key words. galaxies: clusters: general - techniques: spectroscopic

\section{Introduction}

Compact groups (hereafter CGs) are well known systems, ever since the discovery by Edouard Stephan of the first one in 1877 , at a time when we did not even know about the expansion of the universe and about the existence of other galaxies outside our own. These galactic systems drew attention due to their small angular scale; their sizes are comparable to the mean distance between their member galaxies. Physically, we naively classify these systems as having low mass, high projected density, and low velocity dispersion. Galaxy-galaxy interactions (e.g. close tidal encounters) and mergers are therefore likely to dominate their evolution. Until now, however, signs of interactions in CGs selected from the Hickson catalogue, the most widely studied catalogue of such objects (Hickson 1982), were

^ Based on observations obtained at the NTT ESO telescope on La Silla (Chile).

$\star \star$ Table 2, Figs. 2 and 3 are only available in electronic form at http://www. edpsciences.org found to be uncommon and traces of mergers to be rare (Zepf 1993). When present, these features are mainly found in spiraldominated groups.

Subsequent studies by Mendes de Oliveira et al. (1994) showed that, while interactions (i.e. encounters which do not disrupt the galaxies) between galaxies in compact groups are quite common, mergers remain rare, to the level of $6 \%$ of the group galaxies. Addtional studies by Proctor et al. (2004) showed that the stellar population of the early-type member galaxies of nearby, $z \leq 0.03$, compact groups is quite old. This led to doubt the existence of compact groups as physically bound system, but detection of diffuse intra-group matter in $75 \%$ of HCGs (Ponman et al. 1996) confirmed that these are indeed bound, self-gravitating structures. The question then arises about the origin and evolution of compact groups: how can they survive for so long?

Studies of the environment of HCGs (de Carvalho et al. 1997; Ribeiro et al. 1998) have shown that compact groups can 
be divided into three categories, namely: real compact groups, systems composed of a core+halo structure, and sparse groups. Objects belonging to different categories have different surface density profiles, and we observe a propensity for higher activity level in lower velocity dispersion groups. These differences are also reflected in their X-ray emission: groups detected in X-ray have higher galaxy density than groups without detectable X-ray emission. Moreover early type galaxies are more centrally concentrated in X-ray emitting groups than in the non-emitting ones; and finally groups dominated by late type spirals do not show X-ray emission (Zabludoff \& Mulchaey 1998).

The different properties of the group categories identified by de Carvalho et al. (1994, 1997) might be interpreted as an evolutionary scheme in which the groups form in a looser concentration of galaxies, followed by a period of strong evolution with merging episodes. They then settle into a more quiescent phase and finally end up as isolated field ellipticals (Coziol et al. 2004). However, the already cited recent studies by Proctor et al. (2004) and Mendes de Oliveira et al. (2005) find that early type galaxies in compact groups are older than field galaxies of the same type and similar to cluster galaxies. This means that formation of early type galaxies in groups by galaxy-galaxy mergers must have happened a long time ago (of the order of a few Gyrs). On the other hand, compact groups have a very short crossing time, of the order of a few percent of the Hubble time, making it unclear how groups dominated by early type galaxies are observable today, because they should have disappeared a long time ago. A different possibility might be that compact groups are quite young configurations and that we are observing different stages of an ongoing merging process at the same time. If this is true, then we might wonder what a search for compact groups at increasing redshift would produce: would we find an higher number of interacting groups than at present time? Would we find an increase in the activity of the member galaxies? Would we find changes in the group's physical characteristics, like velocity dispersion, mass, radius, and crossing time?

To achieve these goals requires a complete sample of compact groups whose observational and statistical biases are well understood. A new sample of 459 compact group candidates with a median expected redshift of $\sim 0.12$, i.e. 10 times higher than the typical crossing time of present-day compact groups, has been selected with an automatic algorithm applied to the Digitized Second Palomar Observatory Sky Survey II (herafter DPOSS II) galaxy catalogues (Iovino et al. 2003 and de Carvalho et al. 2005). We refer the reader to these cited papers for detailed information on the sample, however, we report here the selection criteria for the sake of completeness:

- richness: $n_{\text {member }} \geq 4$ in the magnitude interval $\Delta \operatorname{mag}_{\text {comp }}=$ $m_{\text {faintest }}-m_{\text {brightest }}$, with the constraint $\Delta$ mag $_{\text {comp }} \leq 2$. Here $n_{\text {member }}$ is the number of member galaxies and $m_{\text {brightest }}$, $m_{\text {faintest }}$ are the magnitude of the brighest and the faintest group members respectively.

- isolation: $R_{\mathrm{isol}} \geq 3 R_{\mathrm{gr}}$, where $R_{\mathrm{isol}}$ is the distance from the centre of the smallest circle encompassing all group galaxies to the nearest non-member galaxy within $0.5 \mathrm{mag}$ of the faintest group member. $R_{\mathrm{gr}}$ is the radius of the smallest circle emcompassing all the galaxies of the group. This criterion avoids finding small aggregates of galaxies within a larger structure, e.g. a cluster.

- compactness: $\mu_{\mathrm{gr}}<\mu_{\text {limit }}$, where $\mu_{g r}$ is the mean surface brightness within the circle of radius $R_{\mathrm{gr}}$ and $\mu_{\text {limit }}=$ $24 \mathrm{mag} \mathrm{arcsec}^{-2}$ in $\mathrm{r}$ band. For comparison, Hickson uses a $\mu_{\text {limit }}=26$ mag $\operatorname{arcsec}^{-2}$, which would have increased the contamination of the sample to $80 \%$, against the current estimate of $27 \%$.

- magnitude of the brightest member galaxy: $16 \leq r \leq 17$.

The magnitude difference criterion is considerably stricter than Hickson's $\left(\Delta \operatorname{mag}_{\text {comp }} \leq 3\right)$, meaning that we have a lower contamination rate, $10 \%$ against $27 \%$, but also reduced completeness.

A first step in exploiting such a sample is to define via spectroscopic follow-up how many groups are indeed bound, selecting subsamples of groups with three/four galaxies sharing the same recession velocity. In addition, the spectroscopic data allow us to estimate the dynamic characteristics of the groups and assess the level of activity in their galaxy population. Here we present the first results from a pilot study carried out at the $3.58 \mathrm{~m}$ New Technology Telescope (NTT) at La Silla Observatory. In the next section we describe the observation and data reduction, in Sect. 3 we present our results and in Sect. 4 our findings.

Throughout the paper, a $\Lambda$ CDM cosmology $\left(\Omega_{\mathrm{M}}=0.3\right.$; $\left.\Omega_{\Lambda}=0.7\right)$ and $H_{0}=67 \mathrm{~km} \mathrm{~s}^{-1} \mathrm{Mpc}^{-1}$ have been used.

\section{The data}

\subsection{Observations and data reduction}

The targets were selected from the published DPOSS II compact group sample by Iovino et al. (2003) on the basis of available observational windows. They represent a fair sample of the total catalogue published in Iovino et al.(2003); in fact a $k$-s test on the observed sample and the complete DPOSS II group catalogue shows that the two populations are the same with a probability of $96.1 \%$. Notice also that the Iovino et al. (2003) sample is a subset (with a few exceptions) of the larger sample of Carvalho et al. (2005), which presents the final complete sample of DPOSS candidate compact groups. These group galaxies were observed with the NTT telescope and the ESO Multi Mode Instrument (hereafter EMMI) in spectroscopic mode in the red arm, equipped with grism \#2 and a slit of $1.5^{\prime \prime}$, under clear/thin cirrus conditions and grey time. The MIT/LL new red detector, a mosaic of 2 CCDs $2048 \times 4096$, was binned by 2 in both spatial and spectral direction, with a resulting dispersion of $3.56 \AA /$ pix, a spatial scale of $0.33^{\prime \prime} /$ pix, an instrumental resolution of $322 \mathrm{~km} \mathrm{~s}^{-1}$, and a wavelength coverage from 3800 to $11000 \AA$. When possible, two or more galaxies were placed together in the slit, whose position angle was constrained by the location of galaxies in the sky and almost never coincided with the parallactic angle. Exposure times varied from $720 \mathrm{~s}$ to $1200 \mathrm{~s}$ per spectrum, and two spectra/target were taken to ensure reliable cosmic ray subtraction. The full log of the observations is given in Table 1. 
Table 1. Observing log.

\begin{tabular}{|c|c|c|c|c|c|}
\hline Group name & $\begin{array}{l}\text { Exp. time } \\
\text { (s) }\end{array}$ & $\overline{\langle\text { seeing }\rangle}$ & 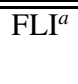 & $\left\langle\overline{\langle X\rangle^{b}}\right.$ & Sky transparency $^{c}$ \\
\hline PCG 100355+190454 & $2 \times 900(\mathrm{~A}, \mathrm{D}) ; 2 \times 720(\mathrm{~B}, \mathrm{C})$ & $0.9^{\prime \prime}$ & 100 & 1.5 & CLR/THN \\
\hline PCG $101345+194541$ & $2 \times 900(\mathrm{~A}, \mathrm{~B}, \mathrm{C}, \mathrm{D})$ & $0.8^{\prime \prime}$ & 30 & 1.6 & CLR/THN \\
\hline PCG 103349+225324 & $2 \times 900(\mathrm{~A}, \mathrm{D}) ; 2 \times 720(\mathrm{~B}, \mathrm{C})$ & $0.9^{\prime \prime}$ & 100 & 1.9 & CLR/THN \\
\hline PCG 103959+274947 & $2 \times 720(\mathrm{~A}, \mathrm{~B}) ; 2 \times 900(\mathrm{C}, \mathrm{D})$ & $1.0^{\prime \prime}$ & 80 & 1.9 & CLR \\
\hline PCG $104338+281711$ & $2 \times 720(\mathrm{~A}, \mathrm{~B}) ; 2 \times 900(\mathrm{C}, \mathrm{D})$ & $1.2^{\prime \prime}$ & 40 & 1.7 & THN \\
\hline PCG $104538+175826$ & $2 \times 720(\mathrm{~A}, \mathrm{~B}) ; 2 \times 900(\mathrm{C}, \mathrm{D})$ & $1.0^{\prime \prime}$ & 90 & 1.5 & CLR/THN \\
\hline PCG $110941+203320$ & $2 \times 900(\mathrm{~A}, \mathrm{~B}, \mathrm{C}, \mathrm{D})$ & $1.2^{\prime \prime}$ & 90 & 1.6 & CLR/THN \\
\hline PCG $114233+140738$ & $2 \times 900(\mathrm{~A}, \mathrm{~B}, \mathrm{C}, \mathrm{D})$ & $0.9^{\prime \prime}$ & 20 & 1.5 & CLR/THN \\
\hline PCG $114333+215356$ & $2 \times 900(\mathrm{~A}, \mathrm{~B}, \mathrm{C}, \mathrm{D})$ & $1.0^{\prime \prime}$ & 100 & 1.6 & CLR/THN \\
\hline PCG $121157+134421$ & $2 \times 900(\mathrm{~A}, \mathrm{~B}, \mathrm{C}, \mathrm{D})$ & $1.2^{\prime \prime}$ & 60 & 1.6 & THN \\
\hline PCG $121252+223519$ & $2 \times 900(\mathrm{~A}, \mathrm{~B}, \mathrm{C}, \mathrm{D})$ & $0.8^{\prime \prime}$ & 100 & 1.7 & CLR/THN \\
\hline PCG $121516+153357$ & $2 \times 720(\mathrm{~A}, \mathrm{~B}, \mathrm{C}, \mathrm{D})$ & $1.0^{\prime \prime}$ & 30 & 1.4 & CLR/THN \\
\hline PCG 130157+191511 & $1 \times 720(\mathrm{~A}, \mathrm{~B}, \mathrm{C}, \mathrm{D})$ & $0.9^{\prime \prime}$ & 70 & 1.5 & CLR \\
\hline PCG $130926+155358$ & $2 \times 900(\mathrm{~A}, \mathrm{~B}, \mathrm{C}, \mathrm{D})$ & $0.7^{\prime \prime}$ & 80 & 1.8 & CLR \\
\hline PCG $145239+275905$ & $2 \times 720(\mathrm{~A}, \mathrm{~B}) ; 2 \times 1200(\mathrm{C}, \mathrm{D})$ & $0.7^{\prime \prime}$ & 90 & 1.9 & CLR \\
\hline PCG $154930+275637$ & $2 \times 900(\mathrm{~A}, \mathrm{~B}, \mathrm{C}, \mathrm{D})$ & $1.2^{\prime \prime}$ & 60 & 1.9 & THN \\
\hline PCG 161754+275834 & $2 \times 900(\mathrm{~A}, \mathrm{~B}, \mathrm{C}, \mathrm{D})$ & $0.7^{\prime \prime}$ & 90 & 1.8 & CLR \\
\hline PCG $170458+281834$ & $2 \times 900(\mathrm{~A}, \mathrm{~B}, \mathrm{C}, \mathrm{D})$ & $1.0^{\prime \prime}$ & 50 & 1.9 & CLR/THN \\
\hline
\end{tabular}

${ }^{a}$ FLI $=$ fraction of illuminated moon.

${ }^{b}$ Average airmass of the group.

${ }^{c} \mathrm{CLR}=$ clear; $\mathrm{THN}=$ thin cirrus.

On-site data reduction was performed using the EMMI spectroscopic quick look tool available at La Silla Observatory (Pompei et al. 2004) and refined later using the MIDAS data reduction package ${ }^{1}$. Our steps include bias subtraction, flatfield correction, wavelength calibration, cosmic ray filtering, sky subtraction, and correction by atmospheric extinction, but no flux calibration, as our nights were not all of photometric quality. Flat field correction was good up to $2 \%$, except in the reddest part of the spectra (redward of $8000 \AA$ ) , where fringing becomes significant (up to $4 \%$ from peak-to-peak variation). As a consequence, absorption lines redward of $8000 \AA$ were not considered for the redshift measurement. A two-dimensional dispersion solution was obtained using the arc frames taken in the afternoon. No arc was taken during the night, since EMMI flexures are less than 1 pixel over the full 360 degrees instrument rotation. A third order polynomial was used for the dispersion direction, while a second order one was employed to correct for the geometrical distortion along the spatial direction. An upper limit of $0.16 \AA$ was found for the rms of the wavelength solution.

Sky subtraction was performed on the two-dimensional rectified spectrum of each target and the rms in the background of the sky subtracted spectra varies from 5 to $10 \%$, which is the worst for nights with a full moon. The atmospheric absorption feature at $\sim 7600 \AA$ was not corrected and any line falling close or in it was not considered for the redshift measurement. The two spectra available for each galaxy were collapsed perpendicular to the dispersion direction, in order to measure the FWHM of the galaxy, on average 4.3". To obtain the final onedimensional, wavelength calibrated galaxy spectrum an extraction window of $3 \times F W H M$ was always used. An exception to

\footnotetext{
${ }^{1}$ Munich Image Data Analysis System, which is developed and maintained by the European Southern Observatory.
}

this rule is represented by two galaxies very close to each other: in this case the biggest non-overlapping window has been chosen, which was never smaller than 4.3".

The two one-dimensional spectra available for each galaxy were averaged together at the end of the reduction, giving an average $S / N$ of $\sim 30$ (grey time) or $\sim 10-15$ (almost full moon) per resolution element at $6000 \AA$.

In some cases, nearby galaxies happened to fall in the slit together with the candidate member galaxies, so their spectra were reduced and extracted following the same procedure used for the target galaxies. Radial velocity standards from the Andersen et al. (1985) paper were observed with the same instrumental set-up used for the target galaxies; in addition to this, we also used galaxy templates with known spectral characteristics and heliocentric velocity available from the literature, i.e. M 32, NGC 7507, and NGC 4111.

\subsection{Redshift measurements}

The IRAF ${ }^{2}$ packages $x c s a o$ and emsao have been used to measure the galaxy redshifts by means of cross-correlation method (Tonry \& Davis 1979), where good results were obtained with galaxy spectra dominated by emission lines or by absorption lines. For spectra dominated by absorption lines, we used galaxy templates and stellar radial velocity standards, while for emission line dominated spectra we used a synthetic template generated by the IRAF package linespec. Starting from a list of the stronger emission lines ( $\mathrm{H} \beta,[\mathrm{OIII}],[\mathrm{OI}], \mathrm{H} \alpha$, [NII], [SII]), the package creates a synthetic spectrum, which is then convolved with the instrumental resolution.

${ }^{2}$ IRAF is distributed by NOAO, which is operated by AURA, Inc, under cooperative agreement with the NSF. 
Following the discussion by Kurtz \& Mink (1998), we performed visual checks of the complete galaxy sample, in order to understand the reliability limit of the automatic redshift determination. This is given by a confidence parameter, $r$, defined for the first time in Tonry \& Davis (1979), which is basically the ratio between the height of the true peak of the correlation function and the average peak of a spurious function (called remainder function in Tonry \& Davis). We found that all redshifts with a confidence parameter $r \geq 5$ are reliable, but measurements with $2.5 \leq r \leq 5$ need to be checked by hand. Measurements with $r \leq 2.5$ are not reliable. All the confirmed member galaxies in our sample had a $r>3.5$.

In some cases emsao failed to correctly identify the emission lines, which happened each time some emission lines were contaminated by underlying absorption. When this happened, we measured the redshift by Gaussian fitting of the strongest emission lines visible and took the average of the results obtained from each line. If two or more lines were blended, the IRAF command deblend within the splot package was used.

The error quoted for the recession velocity is the quadrature sum of two terms: the first is the error in the dispersion solution and the second is the error in the velocity estimate, defined as the scatter in the measurement resulting from the use of different templates, or as the scatter given by the Gaussian fitting of different emission lines. The recession velocity errors varied between 15 and $100 \mathrm{~km} \mathrm{~s}^{-1}$, depending also on the $S / N$ of the target spectrum.

Correction to heliocentric recession velocity values was obtained with the IRAF task rvcorrect in the package noao.rv.

After checking the literature, we found no overlapping data in the $2 \mathrm{dF}$ and $6 \mathrm{dF}$ survey, and only one of our galaxies has a published redshift in Nasa Extragalactic Database (hereafter NED), PCG $114333+215356 \mathrm{~B}$. The quoted redshift is $z=0.130$ (Tovmassian et al. 1999), while the one we measured is $z=0.1319 \pm 0.0002$, i.e. $\sim 600 \mathrm{~km} \mathrm{~s}^{-1}$ difference. On the other hand the published value is quoted there without any errors, and addressing the reader to a forthcoming paper on the optical spectroscopy, which at the time of writing this article, was not yet available. Another comparison can be done with the paper of Miller et al. (2002), where they measure redshifts of galaxies in Abell 0952. Unfortunately, only one galaxy is common to both samples, PGC 101345+194541A, at $\alpha=(10: 13: 45.11)$ and $\delta=(+19: 45: 44.4)$. The two velocity measurements, $33923 \pm 40 \mathrm{~km} \mathrm{~s}^{-1}$ in our case and $33981 \pm$ $52 \mathrm{~km} \mathrm{~s}^{-1}$ for Miller, agree within the experimental errors.

Table 2, which is available only in electronic form, lists the galaxy name (Col. 1), J2000 coordinates from the DPOSS II survey (Cols. 2 and 3), heliocentric radial velocity and velocity error (Cols. 4), type of velocity measurement (from absorption lines "abs", from emission lines "em", from a combination of both "mix"; Col. 5), and the emission lines detected in the spectrum, if any (Col. 6).

\section{Results}

In this section we present the results obtained in our pilot survey. We first discuss group membership and the spectral properties of the member galaxies in detail. We then discuss

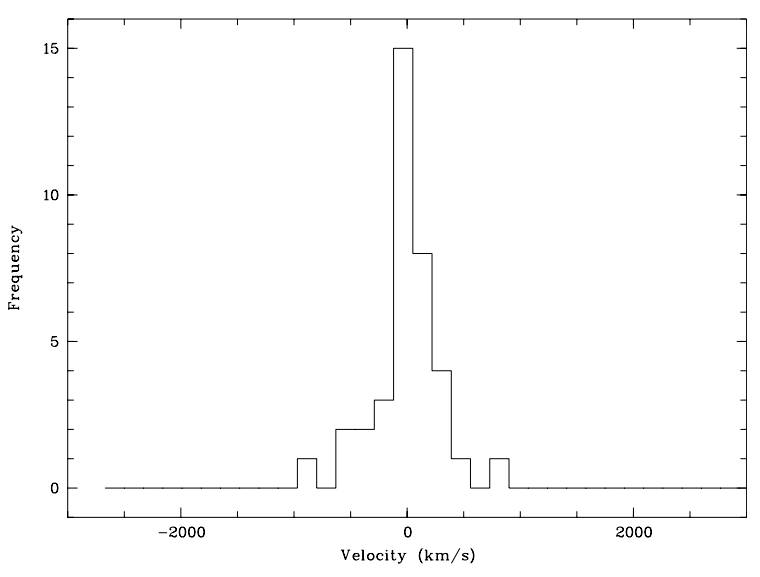

Fig. 1. Distribution of the difference in velocity from the median velocity of the group for all the galaxies cataloged to belong to the same group.

statistical properties of our sample more generally: velocity dispersion, crossing time, $M / L$ ratio and typical size. While it is difficult to produce statistically significant results with only 18 groups, it is nevertheless interesting to examine the trends. We also searched the environment of each group, checking for the possible presence of a nearby known cluster, with the goal of ascertaining whether the group is really isolated or if it belongs to a larger structure.

Finally we note that sometimes other nearby galaxies, close on the sky to our target galaxy but not included as member galaxies in the original group definition, entered in our slit and happened to have a concordant velocity with other members of the group. In order to avoid a contamination of the group selection function or of the group properties, we decided in the following to calculate all the quantities taking into account only the candidate members, as shown on the finding charts on the paper from Iovino et al. (2003).

\subsection{Group membership}

Following the paper by Hickson et al. (1992), we decided to consider as bona fide confirmed groups all the candidates with at least three galaxies, whose relative velocity difference was $\Delta v \leq \pm 1000 \mathrm{~km} \mathrm{~s}^{-1}$ from the median velocity of the group.

The opportunity of such a choice is confirmed by the plot in Fig. 1, which shows the distribution around the median velocity of the group for all galaxies (both concordant and discordant) and originally classified as group members. All the spectroscopically confirmed member galaxies are within $1000 \mathrm{~km} \mathrm{~s}^{-1}$ of the median velocity of the group while the discordant ones are off the scale. This result is very similar to the one found by Hickson for the HCGs catalog.

To calculate the median group velocity and radial velocity dispersion, we used the biweight estimators of location and scale (Beers et al. 1990), which takes all relevant cosmological effects into account. Of a total of 18 candidate compact groups, we found 11 concordant and 7 discordant objects, for a total of $60 \%$ success rate. The radial velocities of our groups range from $c z=23800$ to $44586 \mathrm{~km} \mathrm{~s}^{-1}$, with a median 
Table 3. Classification of the DPOSS II compact groups and neighbouring large scale structures. $n$ represents all galaxies which fulfilled the velocity criteria, irrespective of the original catalogue of group galaxies, while the number quoted in parentheses is the number of concordant galaxies that were originally part of the DPOSS catalog. When a (2+2) is given for $n$ (members), it means that the candidate group is composed of two concordant pairs of galaxies.

\begin{tabular}{|c|c|c|c|c|c|c|c|}
\hline Group name & $\begin{array}{l}\alpha \\
(\mathrm{J} 2000.0)\end{array}$ & $\begin{array}{l}\delta \\
(\mathrm{J} 2000.0)\end{array}$ & $\begin{array}{l}n \\
\text { members }\end{array}$ & $\overline{\langle\mathrm{z}\rangle}$ & Group class & Cluster? & Notes \\
\hline PCG 100355+190454 & 100355 & +190454 & 4 & $0.1076 \pm 0.0002$ & A & - & \\
\hline PCG 101345+194541 & 101345 & +194541 & $9(4)$ & $0.1121 \pm 0.0002$ & B & Abell 0952 & group in the centre of the cluster \\
\hline PCG 103349+225324 & 103349 & +225324 & $2+2$ & - & $\mathrm{C}$ & - & \\
\hline PCG 103959+274947 & 103959 & +274947 & 4 & $0.0989 \pm 0.0001$ & A & - & \\
\hline PCG 104338+281711 & 104338 & +281711 & $2+2$ & - & $\mathrm{C}$ & - & \\
\hline PCG $104538+175826$ & 104538 & +175826 & 2 & - & $\mathrm{C}$ & - & \\
\hline PCG 110941+203320 & 110941 & +203320 & 3 & $0.1389 \pm 0.0002$ & $\mathrm{~B}$ & $\mathrm{~J} 1108+2019$ & $\begin{array}{l}\text { DPOSS II cluster, } z=0.139 \\
\text { group at the outskirt of the cluster }\end{array}$ \\
\hline PCG $114233+140738$ & 114233 & +140738 & 3 & $0.1251 \pm 0.0002$ & B & $\mathrm{J} 1143+1358$ & $\begin{array}{l}\text { DPOSS II cluster, } z=0.120 \\
\text { group at the outskirt of the cluster }\end{array}$ \\
\hline PCG $114333+215356$ & 114333 & +215356 & 4 & $0.1319 \pm 0.0002$ & A & - & SHK 371 \\
\hline PCG $121157+134421$ & 121157 & +134421 & 0 & - & $\mathrm{C}$ & - & \\
\hline PCG $121252+223519$ & 121252 & +223519 & 3 & $0.0850 \pm 0.0002$ & A & - & \\
\hline PCG $121516+153357$ & 121516 & +153357 & 2 & - & $\mathrm{C}$ & - & \\
\hline PCG 130157+191511 & 130157 & +191511 & 3 & $0.0794 \pm 0.0001$ & A & - & \\
\hline PCG $130926+155358$ & 130926 & +155358 & $4(3)$ & $0.1486 \pm 0.0001$ & A & - & \\
\hline PCG 145239+275905 & 145239 & +275905 & $4(3)$ & $0.1255 \pm 0.0001$ & B & Abell 1984 & $\begin{array}{l}\text { group at the outskirt of the cluster } \\
z_{\text {cluster }}=0.124 \\
\text { SHK } 219\end{array}$ \\
\hline PCG $154930+275637$ & 154930 & +275637 & 2 & - & $\mathrm{C}$ & - & \\
\hline PCG 161754+275834 & 161754 & +275834 & 3 & $0.1259 \pm 0.0001$ & A & - & \\
\hline PCG $170458+281834$ & 170458 & +281834 & 2 & - & $\mathrm{C}$ & - & \\
\hline
\end{tabular}

$c z \sim 37534 \mathrm{~km} \mathrm{~s}^{-1}$. These values should be compared to the corresponding ones for the HCGs: radial velocities range from 1380 to $41731 \mathrm{~km} \mathrm{~s}^{-1}$, with a median $c z \sim 8889 \mathrm{~km} \mathrm{~s}^{-1}$.

With the redshift information of each group in hand, we first explored the group environment using NED. We decided to take all the available cluster catalogues into account, with the exception of the Zwicky one, as most of its clusters lack redshift measurements. In our analysis, we also included the DPOSS II cluster catalogue ( $\mathrm{Gal}$ et al. 2003), which is more homogeneous and covers the same depth and area of our group survey, and whose clusters have a reliable photometric redshift estimate.

To find neighboring clusters, we adopted a search radius of $15^{\prime}$, i.e. $\sim 3^{\prime}$ bigger than the Abell radius of a cluster placed at the distance of the our furthest group, $z=0.148$. Once we have a list of possible cluster neighbours, we checked if any of them has a measured redshift. If so, we further refined the search using a redshift criterion by assuming that a group is close to a cluster if the difference in redshift between the two is $\Delta z<$ 0.01 . This corresponds to a velocity difference of $3000 \mathrm{~km} \mathrm{~s}^{-1}$, one order of magnitude above the typical dispersion of compact groups and $\sim 2.5$ times wider than the biggest velocity dispersion measured for clusters $\left(\sim 1200 \mathrm{~km} \mathrm{~s}^{-1}\right.$; Zabludoff et al. 1993).

Accordingly our candidate groups are then divided in three classes: A, B, and C. Candidate groups belonging to class A are confirmed and isolated systems; candidate groups belonging to class B are confirmed but close on the sky to larger structures to which they might be associated. Candidate groups belonging to class $\mathrm{C}$ are all the targets with less than 3 concordant galaxies, and thus not groups according to our definition. Of the 11 confirmed groups, we found seven that we consider class A, while four are close to a cluster. One candidate turned out to be a dense sub-condensation within Abell 0952, while the remaining three are located in the outskirts of known clusters. In Table 3 we show the group name (Col. 1), the coordinates of the centre of the group (Cols. 2, 3), the number of concordant galaxies (Col. 4), the mean redshift of the group (Col. 5), its class (Col. 6) and any neighbour that has been found (Col. 7). No cluster name means none has been found within the search radii used. It is interesting to note that there is only one candidate group with 4 discordant members, PCG 121157+134421.

\subsection{Properties of the group galaxies}

We then analyzed the morphological type of the galaxies in our candidate compact groups. Lacking good quality images, we adopted the same criterium as in Ribeiro et al. (1998), i.e. we assumed that a galaxy has a late morphological type if $E W(\mathrm{H} \alpha)>6.0 \AA^{3}$. The $E W(\mathrm{H} \alpha)$ has been estimated on the wavelength calibrated spectra using the IRAF task splot; to separate the $\mathrm{H} \alpha$ line from the two nearby [NII] lines, we used the deblend task and the results are shown in Table 4 only for groups of class A and B.

We assumed that a group can be considered of late morphological type if at least $50 \%$ of its member galaxies have an $E W(\mathrm{H} \alpha)>6 \AA$, which gives us 5 late type groups from the 11 confirmed targets, see Table 4 . Of the 37 galaxies belonging to the confirmed groups, 13 show emission in $\mathrm{H} \alpha$, for a total of $35 \%$ emitters. No galaxy shows a velocity dispersion

\footnotetext{
${ }^{3}$ We assume here that an emission line has positive $E W$.
} 
Table 4. Morphological classification of the member galaxies for groups of class A and B. $T_{\text {spectrum }}$ is the morphogical type of the group as defined by the percentage of galaxies with $E W(\mathrm{H} \alpha)>6 \AA$.

\begin{tabular}{lllll}
\hline \hline Group name & Members & $n_{-} \mathrm{em}^{a}$ & $T_{\text {spectrum }}$ & $E W(\mathrm{H} \alpha)$ \\
\hline PCG 100355+190454 & 4 & 0 & early & - \\
PCG 101345+194541 & 4 & 0 & early & - \\
PCG 103959+274947 & 4 & 1 & early & A: $6.05 \pm 0.01$ \\
PCG 110941+203320 & 3 & 1 & early & B: $12.98 \pm 0.02$ \\
PCG 114233+140738 & 3 & 1 & early & B: $7.60 \pm 0.02$ \\
PCG 114333+215356 & 4 & 3 & late & A: $22.06 \pm 0.04$ \\
& & & & B: $6.89 \pm 0.02$ \\
PCG 121252+223519 & 3 & 2 & late & A: $24.45 \pm 0.03$ \\
& & & & B: $21.64 \pm 0.06$ \\
PCG 130157+191511 & 3 & 3 & late & A: $36.6 \pm 0.2$ \\
& & & & C: $202 \pm 5$ \\
PCG 130926+155358 & 3 & 1 & early & C: $16.60 \pm 0.05$ \\
PCG 145239+275905 & 3 & 1 & early & B: $7.30 \pm 0.08$ \\
PCG 161754+275834 & 3 & 0 & early & - \\
\hline
\end{tabular}

${ }^{a}$ Number of emitters.

characteristic of a Sy2, but 1 of them is a starburst galaxy, $(E W(\mathrm{H} \alpha) \geq 50 \AA$, Kennicutt \& Kent 1983), PCG 130157+191151C, and another is an HII galaxy, PCG 130157+191151A (see below). This percentage of emission line objects is equal to what has been found for Southern Compact Groups and for Hickson groups, for which the fraction of emission line galaxies is $\sim 35 \%$ of the total (Coziol et al. 2000, 2004).

\subsection{Individual targets}

Here we give a brief description of the individual compact groups selected for this study. The morphological characterisation of the galaxies is based on their measured $E W(\mathrm{H} \alpha)$, as stated in the previous section.

- PCG 100355+190454: this group is composed of 4 early type galaxies in a low background density region. The main galaxy of the group falls very close to a bright star, which could not be separated from the galaxy on the DPOSS II plate. It is the classical example of a group failing the selection criteria and contaminating the sample. If the deblending algorithm had succeeded in separating the galaxy from the nearby bright star, the group would not have been included in the final sample, as none of its member galaxies are brighter than 17 mag in $r$. Even if classified as a class A group, it should be excluded from the final sample.

- PCG 101345+194541: this group deserves special mention as five additional galaxies were observed at the same time as the candidate group members, for a total of nine observed galaxies, all of them are within $1000 \mathrm{~km} \mathrm{~s}^{-1}$ of the mean group velocity. Checking the DSS image, we found that the group actually coincides with a cluster, Abell 0952, whose redshift was measured by Miller et al. (2002), and we can now confirm its location at $z=0.1121 \pm 0.0002$. If we re-measure the velocity dispersion, the crossing time, and the mass and mass-to-light ratio taking all nine galaxies into account, we find substantially different values from those measured using only four galaxies (see next sections): $\log \left(\sigma_{r}\right)=2.76 ; \log \left(H_{0} t_{\mathrm{c}}\right)=-2.03, M=4.11 \times 10^{13} M_{\odot}$, $L=3.23 \times 10^{11} L_{\odot}, M / L=127$. These numbers are indeed more characteristic of a cluster than a compact group; however, they are slightly different from those found by Miller et al. with 10 galaxies. He quotes a velocity dispersion of $512 \mathrm{~km} \mathrm{~s}^{-1}$ against $582 \mathrm{~km} \mathrm{~s}^{-1}$ measured by us. This might be explained by the fact that our observed galaxies form an elongated structure on the sky, while the object observed by the other group are distributed more homogeneously around the centre of the cluster. In fact repeating once more the calculation taking all the observed 19 galaxies (ours + Miller) into account, we find a value of $535 \mathrm{~km} \mathrm{~s}^{-1}$, in good agreement, within the errors, with Miller's estimate. The target has been classified as class B group.

It should be noted that this group is not in the final list of DPOSS compact groups candidates in Carvalho et al. (2005). It is located on a plate whose quality was not good enough to ensure reliable star galaxy separation (explaining a posteriori why such object entered our sample).

- PCG 103349+225324: two pairs of galaxies at discordant redshifts. The brightest galaxy of the nearest pair at $z=$ $0.063 \pm 0.0002$, which is also the main galaxy of the group, shows a disturbed morphology, with asymmetric arms and it is likely to be interacting with galaxy D. The more distant pair, at $z=0.1106 \pm 0.0001$, doesn't show any emission features, but disks are clearly visible in both galaxies. The group has been classified as class $\mathrm{C}$.

- PCG 103959+274947: this group is composed of 4 concordant galaxies, of which only one, the brightest of the group, shows clear spiral arms superimposed on a disk and $\mathrm{H} \alpha+[\mathrm{NII}]$ emission lines. It looks like the classical example of a compact group and has been classified as A.

- PCG 104338+281711: two pairs of galaxies at discordant redshifts. Both galaxies of the closest pair at $z=0.0810 \pm$ 0.0001 , show a disk and $\mathrm{H} \alpha$ emission, while the more distant ones at $z=0.2450 \pm 0.0003$ shows no emission lines. The group has been classified as class $\mathrm{C}$.

- PCG 104538+175826: a disk is clearly visible in galaxies $\mathrm{A}$ and $\mathrm{B}$, but $\mathrm{A}$ has a different redshift from the other galaxies in the group. The disk in B looks asymmetric, and the galaxy is at the same redshift, $z=0.1060 \pm 0.0001$, as $\mathrm{C}$ with which it might be interacting. The group has been classified as class $\mathrm{C}$.

- PCG 110941+203320: a group composed of 1 late type galaxies and two early types; the emission line galaxy, B, has an $\mathrm{H} \alpha$ emission with lower flux than the [NII], probably due to underlying absorption from old stellar population. It is close to the DPOSS cluster J1108+2019 and classified as type B.

- PCG 114233+140738: Galaxy A is the only one with a clearly visible disk in the acquisition image, but it is also the discordant member of the group at $z=0.0990 \pm 0.0001$. Galaxy B shows some emission lines and is classified as late type galaxy, but the other two galaxies are early type 
galaxies. Since galaxy B has a magnitude of 16.7, the group doesn't fail the selection criterion that requires the brightest galaxy to have a magnitude between $16 \leq r \leq 17$. Close to the DPOSS II cluster J1143+1358 this nice triplet of early type galaxies has been classified as B.

- PCG 114333+215356: a group dominated by emission line galaxies. Galaxy B seems to be interacting with another object, but the resolution of the acquisition image doesn't allow better investigation. Its $\mathrm{H} \alpha$ profile shows a narrow peak superimposed on a larger line, probably coming from the other galaxy, which is almost superimposed on galaxy B. A fifth galaxy, E, is also associated with this group, but its faintness did not allow us to obtain a good spectrum. This group was already known in the literature as SHK 371 (Stoll et al. 1997), but its redshift was measured here for the first time. The group belongs to Class A.

- PCG 121157+134421: this group is the only one of our sample with 4 discordant galaxies. Galaxy A, the brightest one, has a clear disk-like morphology similar to galaxy D, which is, however, at a different redshift. The group has been classified as class $C$.

- PCG 121252+223519: this group turned out to be a triplet, with galaxy D a star. The triplet is composed of two late type spiral galaxies, and an early type galaxy, C. Spiral arms are clearly visible in galaxy A, which shows also significant emission in $\mathrm{H} \alpha(E W=24.45 \pm 0.03 \AA)$, while a disk is evident in galaxy $\mathrm{B}$. The group has been classified as class A.

- PCG 121516+153357: nice candidate group of four galaxies, two of which show emission lines. Unfortunately it is composed of one pair (A, D) at $z=0.0980 \pm 0.0001$ and two discordant galaxies. Interestingly enough, galaxy B has a velocity difference of $\sim 1970 \mathrm{~km} \mathrm{~s}^{-1}$ from the AD pair. This is too much for our selection criteria, so the group was not confirmed. However, a DPOSS cluster, J121528+153533 (Gal et al. 2003), with a quoted photometric redshift of 0.147 , is found within the $15^{\prime}$ radius used for our proximity search. The group has been classified as class $\mathrm{C}$.

- PCG 130157+191511: an emission line triplet composed of late type spirals and galaxy B, which is in fact a star. Galaxies $\mathrm{A}$ and $\mathrm{C}$ show disturbed morphology with asymmetric arms and a hint of a tidal tail. Galaxy $\mathrm{C}$ shows strong emission lines characteristics of a starburst galaxy with an $E W(\mathrm{H} \alpha)=202 \pm 5 \AA$, while galaxies $\mathrm{A}$ and D are also strong emitters. This lead us to try a comparison with the most active group in the Hickson sample, HCG 16 (Ribeiro et al., 1996); indeed, the line ratios ([OIII]5007/H $\beta$ ) and ([NII]6584/H $\alpha$ ) of the C and D galaxies are characteristics of starburst nuclear galaxies, while $\mathrm{A}$ is located between HII galaxies and SBNG. If we extract the spectra using an aperture of $1^{\prime \prime}$, i.e. $1.58 \mathrm{kpc}$, all the three galaxies show line ratios characteristics of starburst nuclear galaxies (SBNGs). The group has been classified as class A.

- PCG 130926+155358: again, galaxy B turned out to be a star; one more galaxy which has been included in the slit happened to be at the same redshift of the candidate member galaxies, but it was not taken into account in the calculation of the group parameters. This group is dominated by early type galaxies, since only galaxy $\mathrm{C}$ shows emission in $\mathrm{H} \alpha$ and [NII]. The group has been classified as class A.

- PCG 145239+275905: a group composed of 3 galaxies, one of them showing emission lines. It was already known in the literature as SHK 219 and is at the edge of the Abell cluster 1984. As for SHK 371, the redshift of this group was measured here for the first time. This is a class B group, but it should be noted that this group fails the selection criterion of having the $r$ magnitude of the brightest galaxy between 16 and 17, so it should be excluded from the final sample.

- PCG 154930+275637: Galaxies A, C, and D have a diffuse appearance, and $\mathrm{C}$ and $\mathrm{D}$ seem to be interacting in the acquisition image. Unfortunately only galaxies $\mathrm{A}$ and $\mathrm{D}$ are concordant at $z=0.0760 \pm 0.0002$. The group has been classified as class $\mathrm{C}$.

- PCG 161754+275834: a group dominated by early type galaxies with no emission line detected. The spectrum of galaxy $\mathrm{D}$ is contaminated by strong moon reflection and could not be used. The group has been classified as class A.

- PCG 170458+281834: This group is composed of an interacting pair $(\mathrm{A}, \mathrm{C})$ at $z=0.1640 \pm 0.0002$, while galaxy $\mathrm{B}$ is a star and galaxy $\mathrm{C}$ is a foreground galaxy. All the candidates look like early type galaxies (E/SO) in the acquisition image. The group has been classified as class $\mathrm{C}$.

In Fig. 2, which is available in the electronic version of the paper, we show the acquisition images of the concordant groups (class A and B), while in Fig. 3, also available in the electronic version only, the spectra of their member galaxies are shown. At this point one might wonder about residual contamination of the sample by mis-classified galaxies, i.e. stars. If we assume that our sample is representative of the full compact group DPOSS II survey, we can say that, out of 72 galaxies, we find that 4 have been misidentified and are stars, which means that the residual contamination from incorrect star/galaxy separation is $5.5 \%$. This rate is slightly lower than the quoted error of $9 \%$ in Odewahn et al. (2004); however the authors admit that the $9 \%$ is most likely an overestimation of the real error. Indeed, when looking at their Table 2, it appears that our percentage of wrong star-galaxy separation is comparable to that found by Odewahn et al. for a similar magnitude range.

In the following sections we discuss only the properties of the groups classified as A and B.

\subsection{Internal dynamics and mass estimates}

In Fig. 4 we show the distribution of the velocity dispersion for the observed groups, which is perfectly consistent with that of HCGs and Southern Compact Groups (hereafter SCGs, Iovino 2000), while it is smaller than the average value found for loose groups of galaxies (see for example Eke et al. 2004). The base 10 logarithm of the values measured for the confirmed groups are shown in Col. 3 of Table 5.

To have an idea of how our groups compare to HCGs and to SCGs, we next estimated the crossing time, defined as (Hickson et al. 1992):

$t_{\mathrm{c}}=\frac{4}{\pi} \frac{R}{\sigma_{3 D}}$ 
Table 5. Group dynamical properties. $\sigma_{r}, R$, and $H_{0} t_{\mathrm{c}}$ are expressed in logarithmic values; mass, luminosity, and mass-to-light ratio are given in solar units. The symbols follow Hickson et al. (1992).

\begin{tabular}{cccccccc}
\hline \hline Group name & $\begin{array}{c}\text { Scale } \\
\left(\mathrm{kpc} /{ }^{\prime}\right)\end{array}$ & $\begin{array}{c}\sigma_{r} \\
\left(\mathrm{~km} \mathrm{~s}^{-1}\right)\end{array}$ & $\begin{array}{c}\log (R) \\
(\mathrm{kpc})\end{array}$ & $H_{0} t_{\mathrm{c}}$ & $\begin{array}{c}M \\
M_{\odot}\end{array}$ & $\begin{array}{c}L \\
L_{\odot}\end{array}$ & $M / L$ \\
\hline PCG 100355+190454 & 123.44 & 1.89 & 1.71 & -1.478 & $(1.4 \pm 0.3) \times 10^{12}$ & $(1.4 \pm 0.1) \times 10^{11}$ & 9 \\
PCG 101345+194541 & 136.57 & 2.49 & 1.67 & -2.133 & $(1.3 \pm 0.2) \times 10^{13}$ & $(1.13 \pm 0.09) \times 10^{11}$ & 108 \\
PCG 103959+274947 & 114.70 & 2.36 & 1.46 & -2.203 & $(4.5 \pm 0.8) \times 10^{12}$ & $(7.5 \pm 0.6) \times 10^{10}$ & 55 \\
PCG 110941+203320 & 155.3 & 2.22 & 1.79 & -1.733 & $(6 \pm 2) \times 10^{12}$ & $(1.11 \pm 0.08) \times 10^{11}$ & 48 \\
PCG 114233+140738 & 142.06 & 2.49 & 1.48 & -2.315 & $(1.0 \pm 0.3) \times 10^{13}$ & $(1.4 \pm 0.1) \times 10^{11}$ & 63 \\
PCG 114333+215356 & 147.31 & 1.91 & 1.61 & -1.606 & $(1.3 \pm 0.2) \times 10^{12}$ & $(1.8 \pm 0.1) \times 10^{11}$ & 6 \\
PCG 121252+223519 & 101.06 & 2.01 & 1.47 & -1.845 & $(1.6 \pm 0.4) \times 10^{12}$ & $(1.05 \pm 0.08) \times 10^{11}$ & 13 \\
PCG 130157+191511 & 95.02 & 1.91 & 1.50 & -1.715 & $(7 \pm 2) \times 10^{11}$ & $(6.3 \pm 0.4) \times 10^{10}$ & 10 \\
PCG 130926+155358 & 162.78 & 2.21 & 1.97 & -1.552 & $(8 \pm 2) \times 10^{12}$ & $(1.5 \pm 0.1) \times 10^{11}$ & 47 \\
PCG 145239+275905 & 142.45 & 2.39 & 1.77 & -1.920 & $(1.2 \pm 0.3) \times 10^{13}$ & $(6.9 \pm 0.5) \times 10^{10}$ & 149 \\
PCG 161754+275834 & 141.46 & 2.13 & 1.73 & -1.703 & $(3.4 \pm 0.9) \times 10^{12}$ & $(1.24 \pm 0.09) \times 10^{11}$ & 24 \\
\hline
\end{tabular}

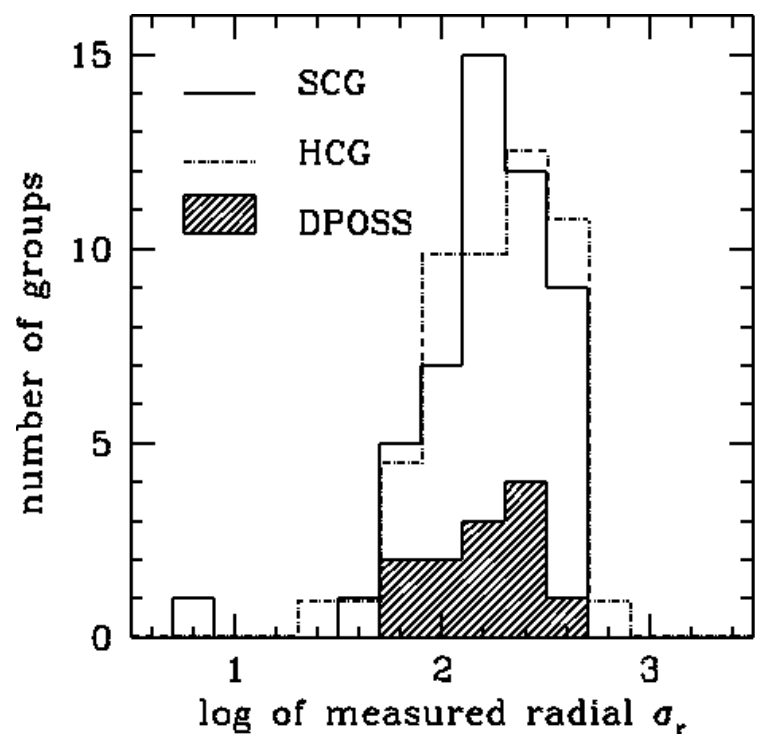

Fig. 4. Distribution of the observed velocity dispersion for the DPOSS groups (hatched area), Southern Compact Groups survey (SCGs, strong line) and Hickson Compact Groups (HCGs, light line).

where $R$ is median of galaxy-galaxy separation and $\sigma_{3 D}$ is the three dimensional velocity dispersion, defined as in Hickson et al. (1992). The logarithm of the crossing time measured for our groups are listed in Col. 5 of Table 5, while the distribution of crossing times is shown in Fig. 5. The median value of $t_{\mathrm{c}}$ is $0.018 H_{0}^{-1}$, in good agreement with what was measured for HCGs, $0.016 H_{0}^{-1}$.

Finally, we provide an estimate of the group mass and the corresponding mass-to-light ratio in the Gunn $r$ filter. To estimate the mass of the groups, we assume that we can use the virial theorem, so that the expression for the mass is:

$M_{\mathrm{V}}=\frac{3 \pi N}{2 G} \frac{\Sigma_{\mathrm{i}} \sigma_{r i}^{2}}{\Sigma_{i<j} 1 / R_{i j}}$

where $R_{i j}$ is the projected separation between galaxies $i$ and $j$, here assumed to be the median length of the two-dimensional galaxy-galaxy separation vector, corrected for cosmological effects. $N$ is the number of concordant galaxies in the system, and $\sigma_{r i}^{2}$ the velocity component along the line of sight of the

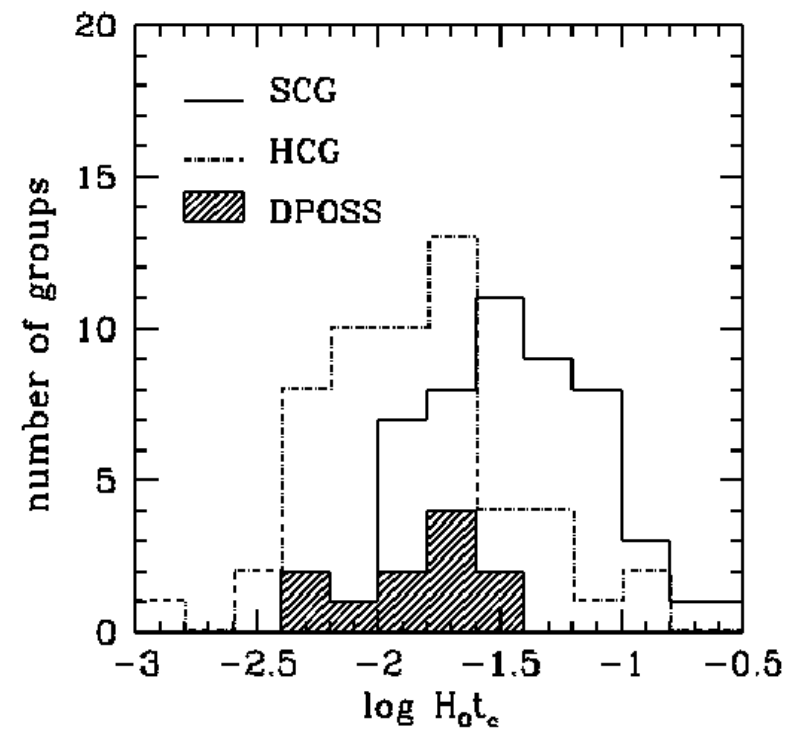

Fig. 5. Distribution of dimensionless crossing time for the confirmed DPOSS groups (hatched area), compared to that obtained for HCGs (light line) and for SCGs (heavy line).

galaxy $i$ with respect to the centre of mass of the group. As observed by Heinsler et al. (1985) and by Perea et al. (1990), the use of the virial theorem produces the best mass estimates, provided that there are no interlopers or projection effects. In case one of these two effects is present, the current values can be considered an upper limit to the real mass. Equation (2) is valid only under the assumption of spherical symmetry and isotropy. Another mass estimate is given by the projected mass estimator, defined as:

$M_{\mathrm{P}}=\frac{f_{\mathrm{P}}}{G N} \Sigma_{i} \sigma_{r i}^{2} R_{i}$

where $R_{i}$ is the projected separation from the centroid of the system, and $f_{\mathrm{P}}$ is a numerical factor depending on the distribution of the orbits around the centre of mass of the system. Assuming a spherically symmetric system for which the Jean's hydrostatic equilibrium applies, we can express $f_{\mathrm{P}}$ in an explicit form (Perea et al. 1990). Since we lacked information 
about the orbit eccentricities, we assumed isotropic orbits and the expression for $M_{\mathrm{P}}$ became:

$M_{\mathrm{P}}=\frac{64}{2 \pi G}\left\langle\sigma_{r}^{2} R\right\rangle$

where again $R$ is the median length of the two-dimensional galaxy-galaxy separation vector.

We calculated both quantities, $M_{\mathrm{V}}$ and $M_{\mathrm{P}}$, and found that $M_{\mathrm{P}}$ is a few percent smaller than $M_{\mathrm{V}}$, but of a comparable order of magnitude. The mass estimate we report in Col. 6 of Table 5 is the average of the two estimators. Errors on the estimate of the mass are largely dominated by the difference between the two estimators, which has been assumed as total error.

To estimate the luminosities, we use the $r$ band magnitudes of the groups obtained by summing up all the flux of the member galaxies as measured on the calibrated DPOSS II plates and also published in Iovino et al. (2003): these are corrected for galactic extinction, and for k-correction, and re-scaled to absolute value. Two different $\mathrm{k}$-corrections have been defined: one for early type galaxies (E-Sa) and another for spirals (Sbc): the $E W(\mathrm{H} \alpha)$ has been used to discriminate in this sense.

As a reference for the solar magnitude, we used the paper by Jorgensen (1994). From stars with a $(B-V) \sim 0.65$ (i.e. the same colour of the sun) it is possible to estimate a colour in$\operatorname{dex}(r-R) \sim 0.354$. Taking $M_{R, \odot}=4.42$ (Binney \& Merrifield 2001), we have $M_{r, \odot}=4.77$. The values for the light and the mass-to-light ratio are shown in Cols. 7 and 8 of Table 5 respectively. Errors on the luminosity were estimated by assuming the maximum error on the photometric calibration of DPOSS plates, i.e. an error of 0.19 mag for an $r$ magnitude of 19 (Gal et al. 2004).

The median value of the $M / L_{r}$ in the sample is 47 . It should be noted, however, that Hickson used $B$-band luminosity, not $r$. If we use the $B$ band luminosity, assuming the transformation (Windhorst et al. 1991),

$B=g+0.51+0.60 \times(g-r)$

and if we take $M_{\mathrm{B}, \odot}=5.48$, we found that the median $M / L_{B}$ is $92 h$, about $50 \%$ bigger than what has been estimated for HCGs. We re-scaled our values to the $H_{0}$ used by Hickson, $100 \mathrm{~km} \mathrm{~s}^{-1}$, with a value of $h=0.67$, to allow easier comparison. The $(g-r)$ colours of the galaxies come from Iovino et al. (2003). Both values are lower than those measured for loose groups, between 200 and $400 h$, but still higher than the value measured for single galaxies in HCGs, $7 h$, see Rubin et al. (1991).

\subsection{Radius distribution}

We can now wonder whether we are indeed looking at compact groups or looser structures. In the first case, we would expect a peak in the distribution around $\sim 50 \mathrm{kpc}$, while in the second a wider distribution is expected.

In Fig. 6, we show the distribution of the physical radius for our confirmed compact groups (classes A and B). We observe a narrow distribution of physical sizes around $\sim 50 \mathrm{kpc}$, with the values greater than $100 \mathrm{kpc}$ given by the very elongated group

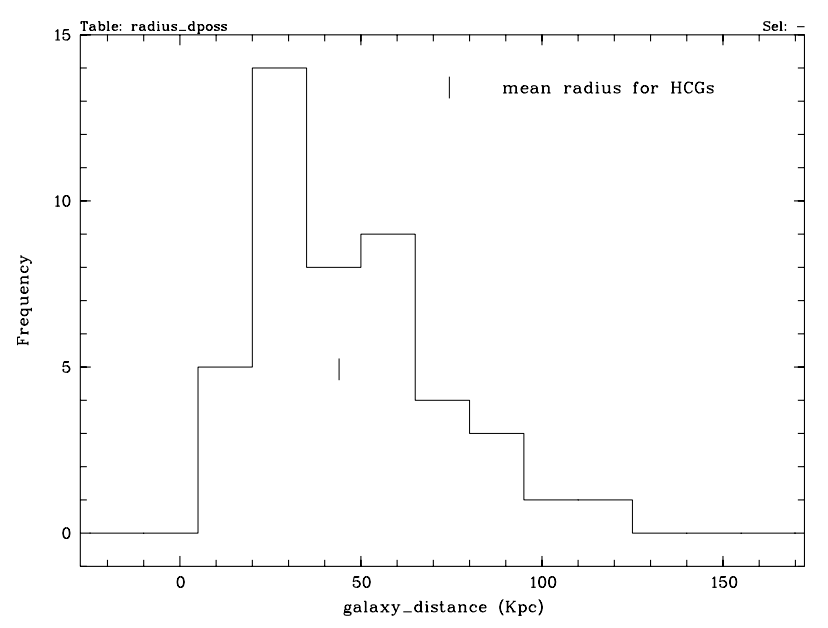

Fig. 6. Distribution of the galaxy-galaxy distance for class A and B groups. The peak of the distribution is consistent with the characteristic physical scale of compact groups, $\sim 50 \mathrm{kpc}$

PCG 130926+155358. This very well agrees with the characteristics physical scale for compact groups, $50 \mathrm{Kpc}$, hence we can be confident within our small number statistics that we are indeed observing compact groups.

\section{Discussion}

Observation and analysis of this small sample of 18 candidate groups have shown several important results of the DPOSS survey:

- Despite the (extremely) low number statistics, the algorithm that identifies compact structures on the DPOSS survey has a success rate of $60 \%$ in identifying dense bound structures on the sky even at an average redshift of 0.2 ; if we consider only isolated compact groups, those objects with 4 spectroscopically concordant galaxies and no neighbour, this rate drops to $22 \%$.

- There is only one candidate group with 4 discordant galaxies: the worst cases, class $\mathrm{C}$ groups, were otherwise always composed of at least one pair of galaxies at a similar redshift.

- The masses of the groups are higher than those found for HCGs, but the median value of $4.5 \times 10^{12} M_{\odot}$ for our sample and $1.5 \times 10^{12} M_{\odot}$ for the HCGs are comparable.

- We are able to identify really compact configurations on the sky, with an average radius of $\sim 50 \mathrm{kpc}$, which is a scale that is usually missed in the current redshift surveys, mainly due to the fiber separation limit.

- About $27 \%$ of the confirmed groups are late type systems.

- Interestingly enough, the most active group of our sample, PCG 130157+191511, contains three star-forming galaxies in it; the only group showing a similar activity in the Hickson's catalog is HCG 16.

As for the first two points, we can ask how this survey work compared with other higher redshift surveys, namely the Las Campanas redshift survey (Allam \& Tucker 1999, 2000; 
Tucker et al. 2000, hereafter LCRS), the Sloan (Lee et al. 2004), and the $2 \mathrm{dF}$ galaxy redshift survey. In the LCRS catalogue it is possible to find two group catalogs: one of compact groups (Allam \& Tucker) and another of loose groups (Tucker et al.). Let us then focus for the moment only on the compact groups catalogue. This catalogue is seriously affected by the fact that the fibers used in the survey have a minimum separation on the sky of $55^{\prime \prime}$, so that it is not possible to measure the individual redshifts of candidate group members whose separation on the sky is less than 55". As a consequence, of the 76 candidate groups of the catalogue, only one has measured redshift for all its members, 23 groups have measured redshifts for two member galaxies, while the rest have just one.

The Sloan survey data do not allow redshift determination for individual group members, again due to the large separation on the sky of the fibers used to carry on the survey: $60^{\prime \prime}$, i.e. $80 h^{-1} \mathrm{kpc}$ at $z \sim 0.1$. Basically, these data are affected by the same problem as LCRS. The identification of a group is made by assigning the same redshift of the brightest galaxy to all galaxies within the fiber limitation circle, or, in the best cases, a group is identified by measuring only two redshifts.

Since we have shown here that only one candidate group out of 18 has all its members with discordant redshifts, the technique used by the Sloan cannot uniquely identify a compact group. In a similar way, the $2 \mathrm{dF}$ survey is affected by limitation due to the fiber diameter; however, many fields have been observed more than once with the fibers in different positions, thus reducing the problem. However, the catalogue created by Merchan \& Zandivarez (2002) covers a wide range in redshift ( 0.003 to 0.25 ), and the mass range and crossing times also include objects that are much more like loose groups than to compact groups.

Finally, one might object that an average redshift of 0.2 is not so very high in an epoch where discovery of $z=3$ targets is common. While this is true, it is important to keep in mind that the projected size of a typical compact group at $z \sim 0.01,1^{\prime}$ corresponds to $13 \mathrm{kpc}$ on the sky, i.e. to a fourth of the typical radius of a compact group $(50 \mathrm{kpc})$. At $z \sim 0.1,1^{\prime}$ is already twice the size of a compact group, and at $z \sim 1$ a compact group is spread over an area of $<20^{\prime \prime}$. To this one must add the increasing fore/background contamination of other objects, making the task of identifying and studying compact groups at higher redshift quite a challenging one.

Summing up, we can reasonably conclude that, of the existing surveys of compact groups at intermediate $z$, the DPOSS II survey and its spectroscopic follow-up will indeed provide, once completed, an unprecedented database for the study of compact groups outside the local universe. The other important question is what the properties of confirmed DPOSS groups tell us. Having only 11 targets makes it difficult to draw firm conclusions. However, we can point out what we would expect if the majority of the DPOSS groups were to show the same characteristics. The fraction of late type galaxies $\left(f_{\mathrm{s}}=0.35\right)$ and the crossing times $\left(H_{0} t_{\mathrm{c}}=0.018\right)$ are similar to those measured for Hickson Compact Groups $\left(f_{\mathrm{s}}=0.49 ; H_{0} t_{\mathrm{c}}=0.016\right)$, while other group characteristics, like mass, and velocity dispersion are also very similar.
With these results in hand, we might wonder whether we have find an answer to our three questions: with increasing redshift, do we find:
- A high number of interacting groups?
- An increase in the number of active groups?
- A change in the average group's physical parameters?

The first two questions are still open because, while we do find a strong star-forming group among 11 targets, we feel that the statistics are still too poor to extrapolate this finding to the full sample. Ongoing observations at La Silla on the southern part of the sample will help to improve the statistics. For the last question, the answer seems to be no, because our results point toward no evolution for compact groups up to $z=0.12$, which is the median redshift of our observed sample.

This indeed looks puzzling, since a redshift of $z=0.12$ corresponds to a look-back time of $1.56 \mathrm{Gyr}$, while our compact groups should have dissolved in $0.21 \mathrm{Gyrs}$, given their median crossing time of $t_{\mathrm{c}}=0.018 \mathrm{H}_{0}^{-1}$. This could mean that either we are looking at a different set of groups from the one we observe in the nearby Universe, e.g. groups that have already merged by the present time, leaving a field elliptical or a substructure within a cluster, or there is something that stabilizes the groups extending their lifetime.

This seems to agree well with numerical models that favour an early formation of a common, massive halo within which individual galaxies form (Gomez-Flechoso \& Dominguez-Tenreiro 2001). This model, unlike the one proposed by Atahnassoula \& Makino (1997), predicts a central concentration for the common halo, in agreement with the observations. Moreover, according to this model, the galaxy interactions perturb the global halo potential and become significant in changing the group only if they are comparable to the global field of force of the halo. This scenario seems consistent with previous findings that individual galaxy properties within the group do not correlate with the group global properties. It must be kept in mind, however, that active groups with a low velocity dispersion, like those present in the Southern Compact Group survey, are still not explained well by this scenario, so that a more detailed investigation is needed to understand the nature of compact groups completely.

\section{Conclusions}

Our pilot study of compact groups at intermediate redshift has shown that the confirmed candidates have very similar properties to those observed for Hickson Compact Groups and that no significant evolution can be detected up to $z \sim 0.12$. This finding agrees with models predicting an early formation of a massive, common halo, within which the individual galaxies form and evolve. Such models, however, are still unable to explain the low velocity dispersion, high activity level groups found in the nearby universe.

Our results suggest that the DPOSS sample, once full redshift information for its members becomes available, will provide a reference sample for studying the properties of compact groups beyond the local universe. 
Acknowledgements. It is a pleasure to thank the La Silla Science Operation team for their help during the observations and daytime support. R.R.dC. would like to thank Roy Gal for several insightful discussions on the subject.

This research made use of the NASA/IPAC Extragalactic Database (NED), which is operated by the Jet Propulsion Laboratory, California Institute of Technology, under contract with the National Aeronautics and Space Administration.

\section{References}

Allam, S. S., \& Tucker, D. L. 1999, ApJ, 522, L89

Allam, S. S., \& Tucker, D. L. 2000, AN, 321, 101

Andersen, J., Nordstrom, B., Ardeberg, A., et al. 1985, A\&AS, 59, 15

Athanassoula, E., Makino, J., \& Bosma, A. 1997, MNRAS, 286, 825

Beers, T., Flynn, K., \& Gebhardt, K. 1990, AJ, 100, 32

Binney, J., \& Merrifield, M. 2001 Galactic astronomy (Princeton University Press)

Coziol, R., de Carvalho, R. R., Capelato, H. V, \& Ribeiro, A. L. B. 1998a, ApJ, 506, 545

Coziol, R., Ribeiro, A. L. B., Capelato, H. V., \& de Carvalho, R. R. 1998b, ApJ, 493, 563

Coziol, R., Iovino, A., \& de Carvalho, R. R. 2000, AJ, 120, 47

Coziol, R., Brinks, E., \& Bravo-Alfaro, H. 2004, AJ, 128, 68

de Carvalho, R. R., Ribeiro, A., \& Zepf, S. 1994, ApJS, 93, 47

de Carvalho, R. R., Ribeiro, A., Capelato, H., \& Zepf, S. 1997, ApJS, 110,1

de Carvalho, R. R., Goncalves, T. S., Iovino A., et al. 2005 [arXiv: astro-ph/0504217]

Diaferio, A., Geller, M., \& Ramella, M. 1994, AJ, 107, 868

Eke, V. R., Baugh, C. M., Cole, S. et al. 2004, MNRAS, 348, 866

Gal, R. R., de Carvalho, R. R., \& Lopes, P. A. A., et al. 2003, AJ, 125, 2064

Gal, R. R., de Carvalho, R. R., Odewahn, S. C., et al. 2004, AJ, 128, 3082

Gomez-Flechoso, M. A., \& Dominguez-Tenreiro, R. 2001 ApJ, 549, L187

Heisler, J., Tremaine, S., \& Bahcall, J. 1985, ApJ, 298, 8

Hickson, P. 1982, ApJ, 255, 382
Hickson, P., Mendes de Oliveira, C., Huchra, J. P., \& Palumbo, G. G. C. 1992, ApJ, 399, 353

Iovino, A. 2000, ASP, 174, 25

Iovino, A. 2002, AJ, 124, 2471

Iovino, A., de Carvalho R. R., Gal, R. R., et al. 2003, AJ, 125, 1660

Jorgensen, I., 1994, PASP, 106, 967

Kennicutt, R., \& Kent, S. M. 1983, AJ, 88, 1094

Kurtz, M. J., \& Mink, D. J. 1998, PASP, 110, 934

Lee, B. C, Allam, S. S., Tucker, D. L., et al. 2004, AJ, 127, 1811

Mendes de Oliveira, C., \& Hickson, P. 1994, ApJ, 427, 684

Mendes de Oliveira, C., Coelho, P., Gonzalez, J. J., \& Barbuy, B. 2005, AJ, 130, 55

Merchán, M. E., \& Zandivarez, A. 2002, MNRAS, 335, 216

Miller, C. J., Krughoff, K. S, Batuski, D. J., \& Mill, J. M. 2002, AJ, 124, 1918

Odewahn, S. C., de Carvalho, R. R., Gal, R. R., et al. 2004, AJ, 128, 3092

Perea, J., del Olmo, A., \& Moles, M. 1990, A\&A, 237, 319

Pompei, E., et al. 2004 ESO-Messenger, 116, 16

Ponman, T. J., Bourner, P. D. J., Ebeling, H., \& Bohringer, H. 1996, MNRAS, 283, 690

Proctor, R, Forbes, D. A., Hau, G. K. T., et al. 2004, MNRAS, 349, 1381

Ribeiro, A., de Carvalho, R. R., Coziol, R., Capelato, H., \& Zepf, S. E. 1996, ApJ, 463, 5

Ribeiro, A., de Carvalho, R. R., Capelato, H., \& Zepf, S. E. 1998, ApJ, 497, 72

Rubin, V. C., Hunter, D. A., \& Ford, W. K. 1991, ApJS, 76, 153

Stoll, D., Tiersch, H., \& Cordis, L. 1997, AN, 318, 89

Tonry, J., \& Davis, M. 1979, AJ, 84, 1511

Tovmassian, H. M., \& Chavushyan, V. H. 1999, ApJ, 523, 87

Tucker, D. L, Oemler, A., Jr., Hashimoto, Y., et al. 2000, ApJS, 130, 237

Windhorst, R. A., Burstein, D., Mathis, D. F., et al. 1991, ApJ, 380, 362

Zabludoff, A. I., Geller, M., Huchra, J., \& Vogeley, M. 1993, AJ, 106, 1273

Zabludoff, A. I., \& Mulchaey, J. S. 1998, ApJ, 496, 39

Zepf, S. E. 1993, ApJ, 407, 488 


\section{Online Material}


E. Pompei et al.: DPOSS II compact groups survey, Online Material $p 2$

Table 2. Observed compact groups candidates from the DPOSS survey. Column 5 lists the kind of spectrum for each galaxy: abs means a spectrum which is dominated by absorption lines; em means a spectrum dominated by emission lines and mix a spectrum where both emission and absorption lines are present. The most significant emission lines have been listed for the observed galaxies.

\begin{tabular}{|c|c|c|c|c|c|}
\hline Galaxy name & $\begin{array}{c}\alpha \\
(\mathrm{J} 2000.0)\end{array}$ & $\begin{array}{c}\delta \\
(\mathrm{J} 2000.0)\end{array}$ & $\begin{array}{c}c z \\
\left(\mathrm{~km} \mathrm{~s}^{-1}\right)\end{array}$ & Line & Emission lines \\
\hline PCG 100355+190454A & 100354.22 & +19052.11 & $32234 \pm 75$ & abs & - \\
\hline PCG 100355+190454B & 100356.92 & +19052.11 & $32494 \pm 56$ & abs & - \\
\hline PCG $100355+190454 C$ & 100355.70 & +190455.09 & $32294 \pm 42$ & abs & - \\
\hline PCG 100355+190454D & 100353.61 & +190447.21 & $32355 \pm 42$ & abs & - \\
\hline PCG 101345+194541A & 101345.16 & +194544.28 & $33923 \pm 40$ & abs & - \\
\hline PCG 101345+194541B & 101346.87 & +194530.71 & $33254 \pm 41$ & abs & - \\
\hline PCG 101345+194541C & 101344.87 & +194551.52 & $33378 \pm 32$ & abs & - \\
\hline PCG 101345+194541D & 101345.84 & +194537.61 & $34033 \pm 82$ & abs & - \\
\hline PCG 103349+225324A & 103348.96 & +225344.60 & $18775 \pm 68$ & em & [OII], $\mathrm{H} \beta,[\mathrm{OIII}], \mathrm{H} \alpha,[\mathrm{NII}],[\mathrm{SII}]$ \\
\hline PCG 103349+225324B & 103349.50 & +225314.43 & $32949 \pm 59$ & abs & - \\
\hline PCG 103349+225324C & 103350.26 & +225315.68 & $33413 \pm 56$ & abs & - \\
\hline PCG 103349+225324D & 103347.75 & +225334.15 & $18869 \pm 70$ & em & $\mathrm{H} \alpha,[\mathrm{NII}],[\mathrm{SII}]$ \\
\hline PCG 103959+274947A & 103959.23 & +274935.72 & $29900 \pm 20$ & em & $\mathrm{H} \alpha,[\mathrm{NII}]$ \\
\hline PCG 103959+274947B & 103958.52 & +274944.98 & $29863 \pm 32$ & abs & - \\
\hline PCG 103959+274947C & 103959.13 & +274959.48 & $30503 \pm 28$ & abs & - \\
\hline PCG 103959+274947D & 103958.90 & +274941.88 & $29764 \pm 52$ & abs & - \\
\hline PCG 104338+281711A & 104338.19 & +281721.23 & $23339 \pm 29$ & em & $\mathrm{H} \alpha,[\mathrm{NII}],[\mathrm{SII}]$ \\
\hline PCG 104338+281711B & 104338.97 & +281713.09 & $25318 \pm 85$ & em & $\mathrm{H} \alpha,[\mathrm{NII}],[\mathrm{SII}]$ \\
\hline PCG 104338+281711C & 104339.40 & +281705.49 & $73583 \pm 69$ & abs & - \\
\hline PCG 104338+281711D & 104337.83 & +281706.68 & $73525 \pm 77$ & abs & - \\
\hline PCG 104538+175826A & 104539.91 & +175831.48 & $21687 \pm 36$ & abs & - \\
\hline PCG 104538+175826B & 104538.98 & +17587.86 & $31751 \pm 50$ & abs & - \\
\hline PCG 104538+175826C & 104537.78 & +175817.55 & $31654 \pm 49$ & abs & - \\
\hline PCG 104538+175826D & 104537.90 & +175845.16 & $39071 \pm 62$ & abs & - \\
\hline PCG 110941+203320A & 110942.65 & +203329.67 & $34956 \pm 60$ & em & $\mathrm{H} \alpha,[\mathrm{NII}],[\mathrm{SII}]$ \\
\hline PCG 110941+203320B & 110939.79 & +203311.20 & $41593 \pm 60$ & em & $\mathrm{H} \alpha,[\mathrm{NII}]$ \\
\hline PCG 110941+203320C & 110941.06 & +203335.53 & $41511 \pm 55$ & abs & - \\
\hline PCG 110941+203320D & 110940.29 & +203313.28 & $42005 \pm 60$ & abs & - \\
\hline PCG 114233+140738A & 114233.84 & +140751.56 & $29860 \pm 37$ & abs & - \\
\hline PCG 114233+140738B & 114232.39 & +140725.61 & $37285 \pm 90$ & em & {$[\mathrm{OII}], \mathrm{H} \alpha,[\mathrm{NII}]$} \\
\hline PCG 114233+140738C & 114233.78 & +140727.09 & $37362 \pm 40$ & abs & - \\
\hline PCG $114233+140738 D$ & 114232.83 & +140728.27 & $38136 \pm 45$ & abs & - \\
\hline PCG 114333+215356A & 114333.15 & +215350.32 & $39806 \pm 20$ & em & {$[\mathrm{OII}], \mathrm{H} \beta,[\mathrm{OIII}],[\mathrm{OI}], \mathrm{H} \alpha,[\mathrm{NII}]$} \\
\hline PCG 114333+215356B & 114332.87 & +215405.44 & $39586 \pm 40$ & em & $\mathrm{H} \alpha,[\mathrm{NII}]$ \\
\hline PCG 114333+215356C & 114332.93 & +215356.37 & $39558 \pm 23$ & $\mathrm{em}$ & {$[\mathrm{OII}], \mathrm{H} \alpha,[\mathrm{NII}]$} \\
\hline PCG $114333+215356 \mathrm{D}$ & 114333.53 & +215421.46 & $39531 \pm 72$ & abs & - \\
\hline PCG 121157+134421A & 121158.54 & +134429.57 & $24217 \pm 15$ & $\operatorname{mix}$ & $\mathrm{H} \alpha,[\mathrm{NII}]$ \\
\hline PCG 121157+134421B & 121157.30 & +134413.24 & $52149 \pm 96$ & abs & - \\
\hline PCG 121157+134421C & 121157.78 & +134416.33 & $20574 \pm 98$ & abs & - \\
\hline PCG $121157+134421 \mathrm{D}$ & 121158.69 & +134419.54 & $87724 \pm 100$ & abs & - \\
\hline PCG 121252+223519A & 121253.31 & +223526.55 & $25502 \pm 74$ & em & [OIII], [OI], H $\alpha,[\mathrm{NII}]$ \\
\hline PCG 121252+223519B & 121251.93 & +223530.08 & $25457 \pm 80$ & $\mathrm{em}$ & $\mathrm{H} \alpha,[\mathrm{NII}]$ \\
\hline PCG 121252+223519C & 121252.42 & +223514.28 & $25770 \pm 57$ & abs & - \\
\hline PCG 121252+223519D & 121252.55 & +223506.94 & STAR! & - & - \\
\hline PCG 121516+153357A & 121516.30 & +153425.29 & $29456 \pm 47$ & abs & - \\
\hline PCG 121516+153357B & 121515.07 & +153350.91 & $314324 \pm 50$ & abs & - \\
\hline PCG 121516+153357C & 121514.44 & +153357.85 & $24477 \pm 50$ & $\operatorname{mix}$ & $\mathrm{H} \alpha,[\mathrm{NII}]$ \\
\hline PCG 121516+153357D & 121515.71 & +153334.89 & $29535 \pm 47$ & $\operatorname{mix}$ & $\mathrm{H} \alpha,[\mathrm{NII}],[\mathrm{SII}]$ \\
\hline PCG 130157+191511A & 130156.80 & +191454.93 & $23750 \pm 30$ & em & {$[\mathrm{OII}], \mathrm{H} \beta,[\mathrm{OIII}],[\mathrm{OI}], \mathrm{H} \alpha,[\mathrm{NII}],[\mathrm{SII}]$} \\
\hline PCG 130157+191511B & 130155.98 & +191506.26 & STAR! & - & - \\
\hline PCG 130157+191511C & 130157.72 & +191511.27 & $23931 \pm 22$ & em & {$[\mathrm{OII}], \mathrm{H} \gamma, \mathrm{H} \beta,[\mathrm{OIII}],[\mathrm{OI}], \mathrm{H} \alpha,[\mathrm{NII}],[\mathrm{SII}]$} \\
\hline
\end{tabular}


Table 2. continued.

\begin{tabular}{|c|c|c|c|c|c|}
\hline Galaxy name & $\begin{array}{c}\alpha \\
(\mathrm{J} 2000.0)\end{array}$ & $\begin{array}{c}\delta \\
(\mathrm{J} 2000.0)\end{array}$ & $\begin{array}{c}c z \\
\left(\mathrm{~km} \mathrm{~s}^{-1}\right)\end{array}$ & Line & Emission lines \\
\hline PCG 130157+191511D & 130158.24 & +191520.38 & $23721 \pm 43$ & em & {$[\mathrm{OII}], \mathrm{H} \gamma, \mathrm{H} \beta,[\mathrm{OIII}],[\mathrm{OI}], \mathrm{H} \alpha,[\mathrm{NII}],[\mathrm{SII}]$} \\
\hline PCG 130926+155358A & 130926.89 & +155419.48 & $44817 \pm 20$ & abs & - \\
\hline PCG 130926+155358B & 130926.26 & +155407.67 & STAR! & - & - \\
\hline PCG 130926+155358C & 130926.44 & +155412.20 & $44406 \pm 50$ & $\operatorname{mix}$ & $\mathrm{H} \alpha,[\mathrm{NII}]$ \\
\hline PCG 130926+155358D & 130926.91 & +155337.82 & $44896 \pm 20$ & abs & - \\
\hline PCG 145239+275905A & 145239.07 & +275850.42 & $35907 \pm 35$ & $\operatorname{mix}$ & $\mathrm{H} \alpha,[\mathrm{NII}]$ \\
\hline PCG 145239+275905B & 145240.31 & +275848.18 & $38188 \pm 65$ & $\operatorname{mix}$ & $\mathrm{H} \alpha,[\mathrm{NII}]$ \\
\hline PCG 145239+275905C & 145239.47 & +275922.67 & $38019 \pm 54$ & abs & - \\
\hline PCG 145239+275905D & 145238.65 & +275901.67 & $37490 \pm 71$ & abs & - \\
\hline PCG 154930+275637A & 154930.29 & +275620.47 & $22910 \pm 18$ & $\operatorname{mix}$ & $\mathrm{H} \alpha,[\mathrm{NII}]$ \\
\hline PCG 154930+275637B & 154929.11 & +275641.79 & $36450 \pm 80$ & abs & - \\
\hline PCG 154930+275637C & 154931.49 & +275640.99 & $28381 \pm 30$ & $\operatorname{mix}$ & $\mathrm{H} \alpha,[\mathrm{NII}],[\mathrm{SII}]$ \\
\hline PCG 154930+275637D & 154931.41 & +275646.83 & $22893 \pm 20$ & $\operatorname{mix}$ & $\mathrm{H} \alpha,[\mathrm{NII}]$ \\
\hline PCG 161754+275834A & 161755.29 & +275832.01 & $37779 \pm 35$ & abs & - \\
\hline PCG 161754+275834B & 161754.17 & +2758 13.98 & $37748 \pm 36$ & abs & - \\
\hline PCG 161754+275834C & 161754.86 & +275812.61 & $38111 \pm 30$ & abs & - \\
\hline PCG 161754+275834D & 161755.72 & +275841.48 & $\mathrm{~N} / \mathrm{A}$ & - & strong moon reflection in the spectrum \\
\hline PCG 170458+281834A & 170457.36 & +281833.81 & $49131 \pm 56$ & abs & - \\
\hline PCG 170458+281834B & 170458.02 & +281809.29 & STAR! & & - \\
\hline PCG 170458+281834C & 170459.77 & +281842.95 & $24318 \pm 42$ & $\operatorname{mix}$ & $\mathrm{H} \alpha,[\mathrm{NII}]$ \\
\hline PCG 170458+281834D & 170456.59 & +281850.58 & $49431 \pm 59$ & abs & - \\
\hline
\end{tabular}


E. Pompei et al.: DPOSS II compact groups survey, Online Material $p 4$
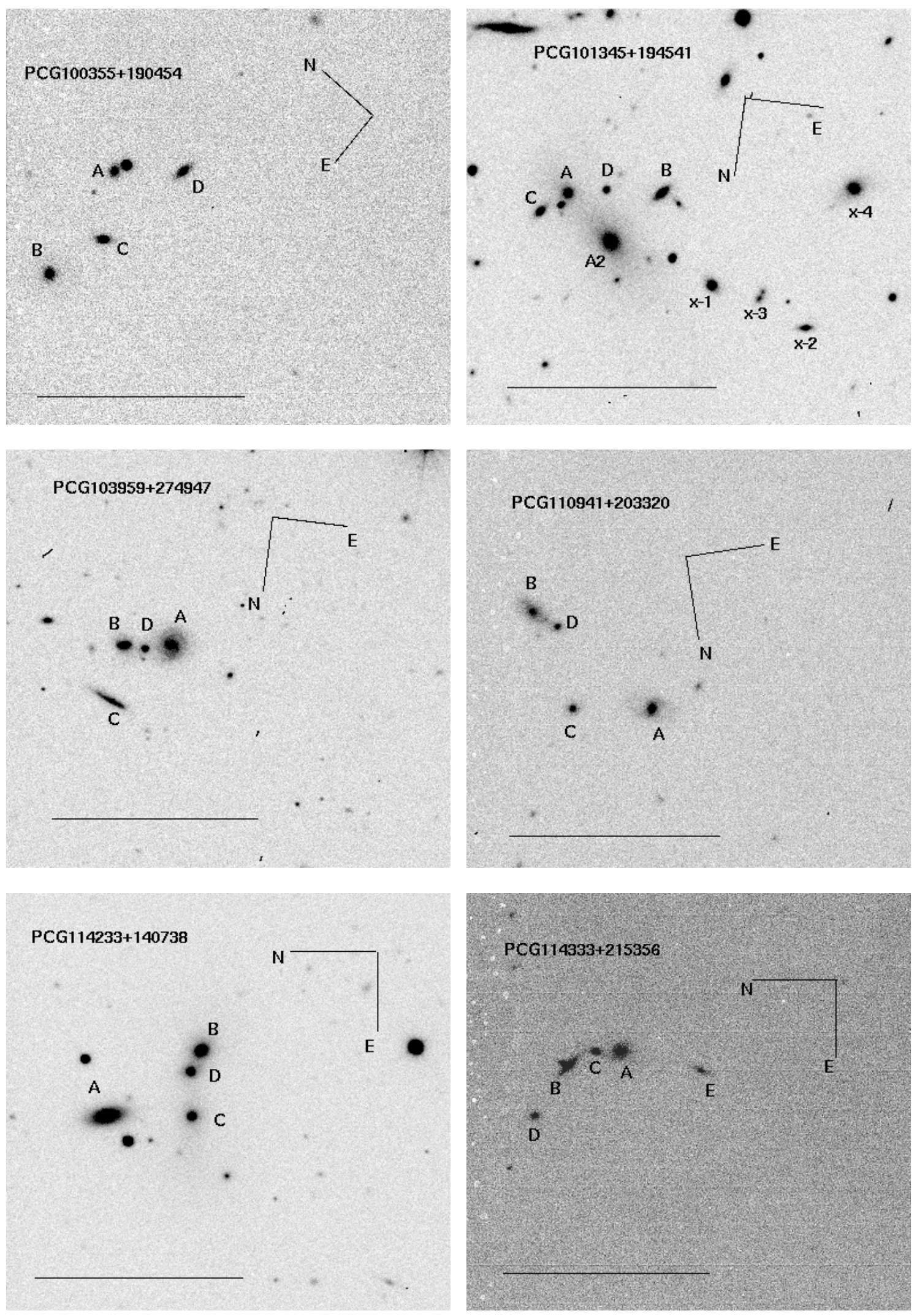

Fig. 2. Acquisition images of the concordant groups. The orientation is explicitly given for each frame. The individual galaxies are labelled as in the DPOSS catalogue. Galaxies labelled with an $\mathrm{x}$ followed by a number are objects which fell in the slit along the target galaxies and turned out to be concordant with the DPOSS targets. The solid line at the bottom of each image corresponds to $1^{\prime}$. 
E. Pompei et al.: DPOSS II compact groups survey, Online Material p 5
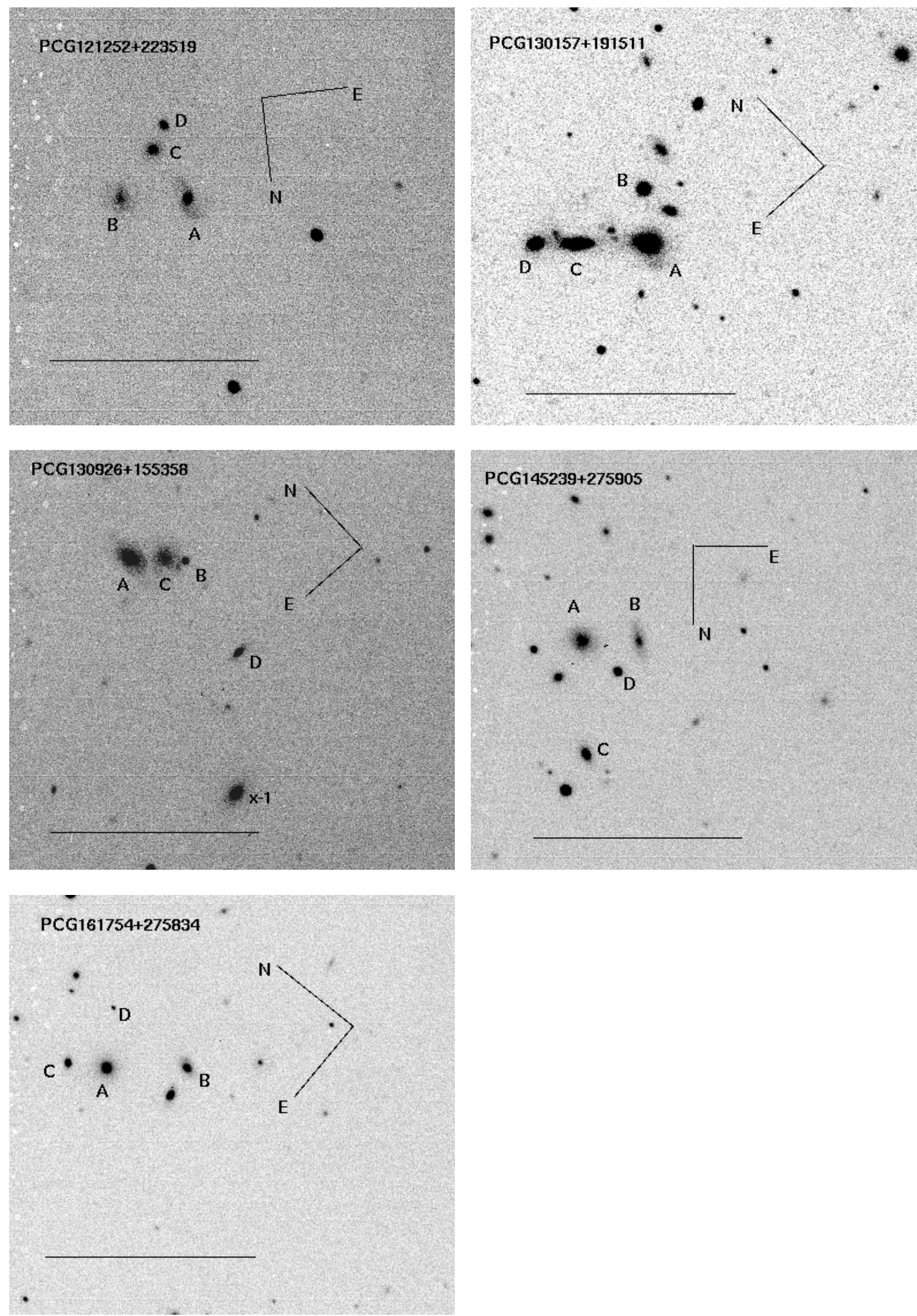

Fig. 2. continued. 
E. Pompei et al.: DPOSS II compact groups survey, Online Material $p 6$
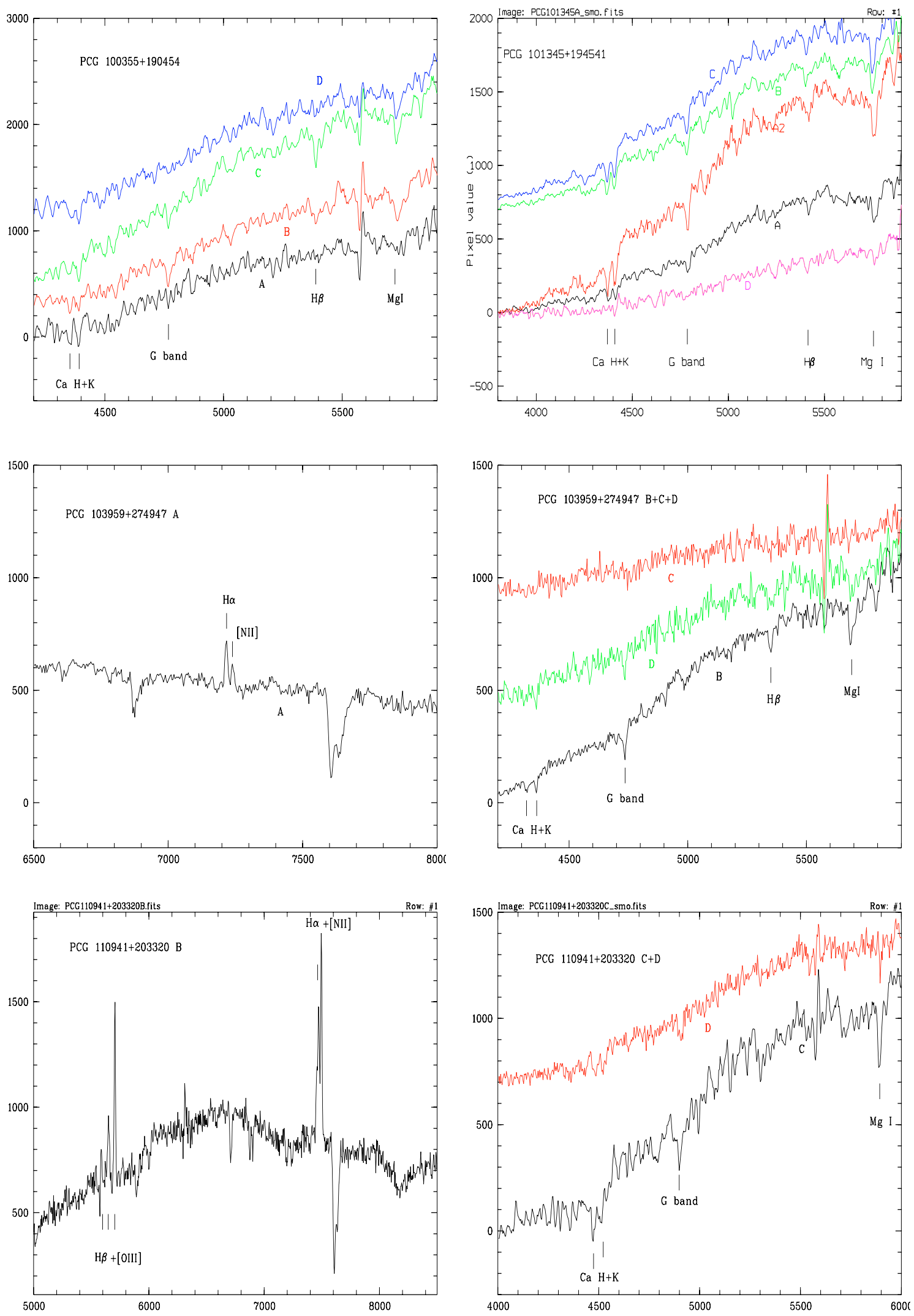

Fig. 3. Spectral atlas of the concordant member galaxies for groups of class A and B. On the $x$ axis there is the wavelength, while on the $y$ axis there are the number counts (ADU). The spectra have been shifted an arbitrary amount for display purpose. 
E. Pompei et al.: DPOSS II compact groups survey, Online Material p 7
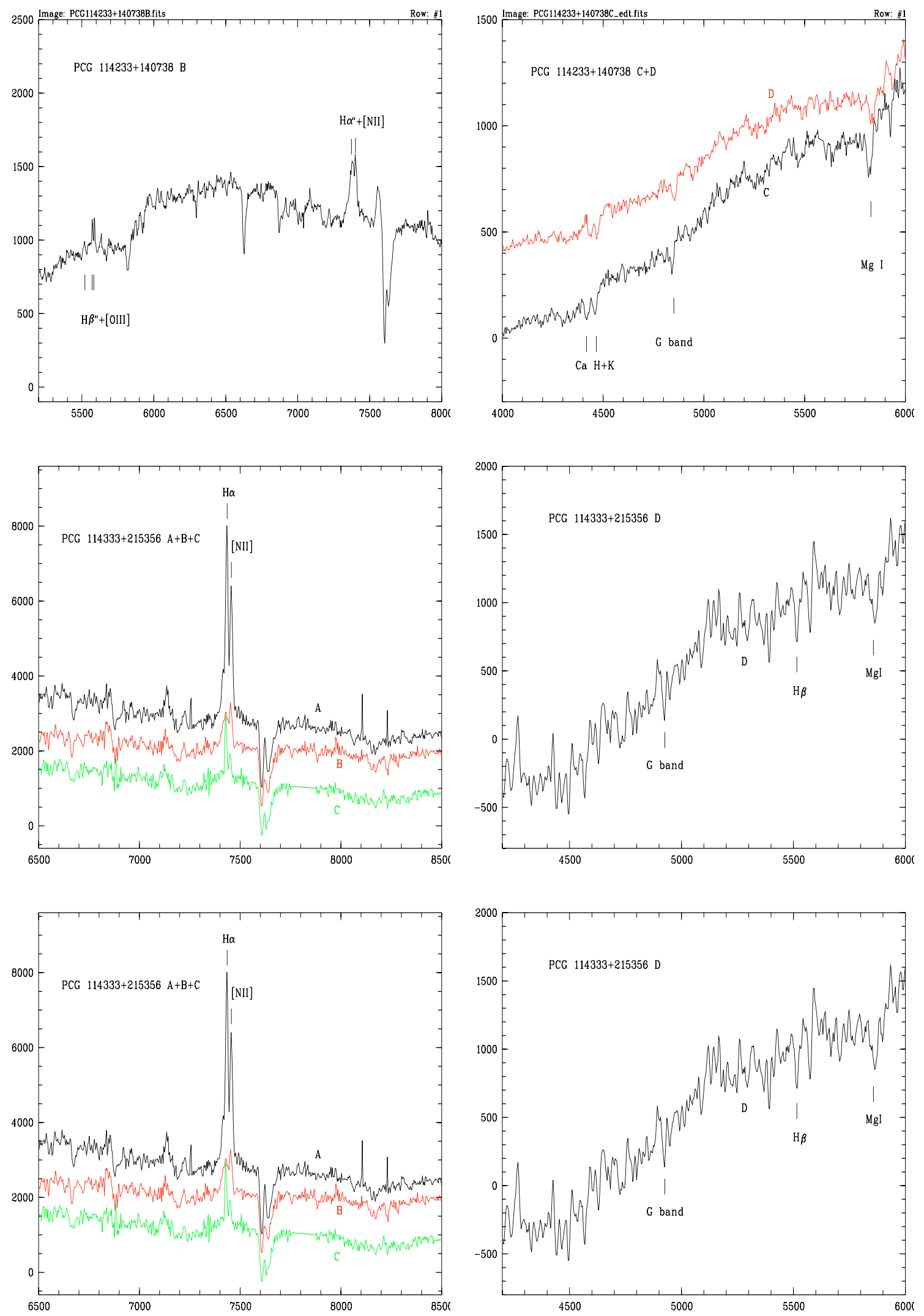

Fig. 3. continued. 
E. Pompei et al.: DPOSS II compact groups survey, Online Material $p 8$
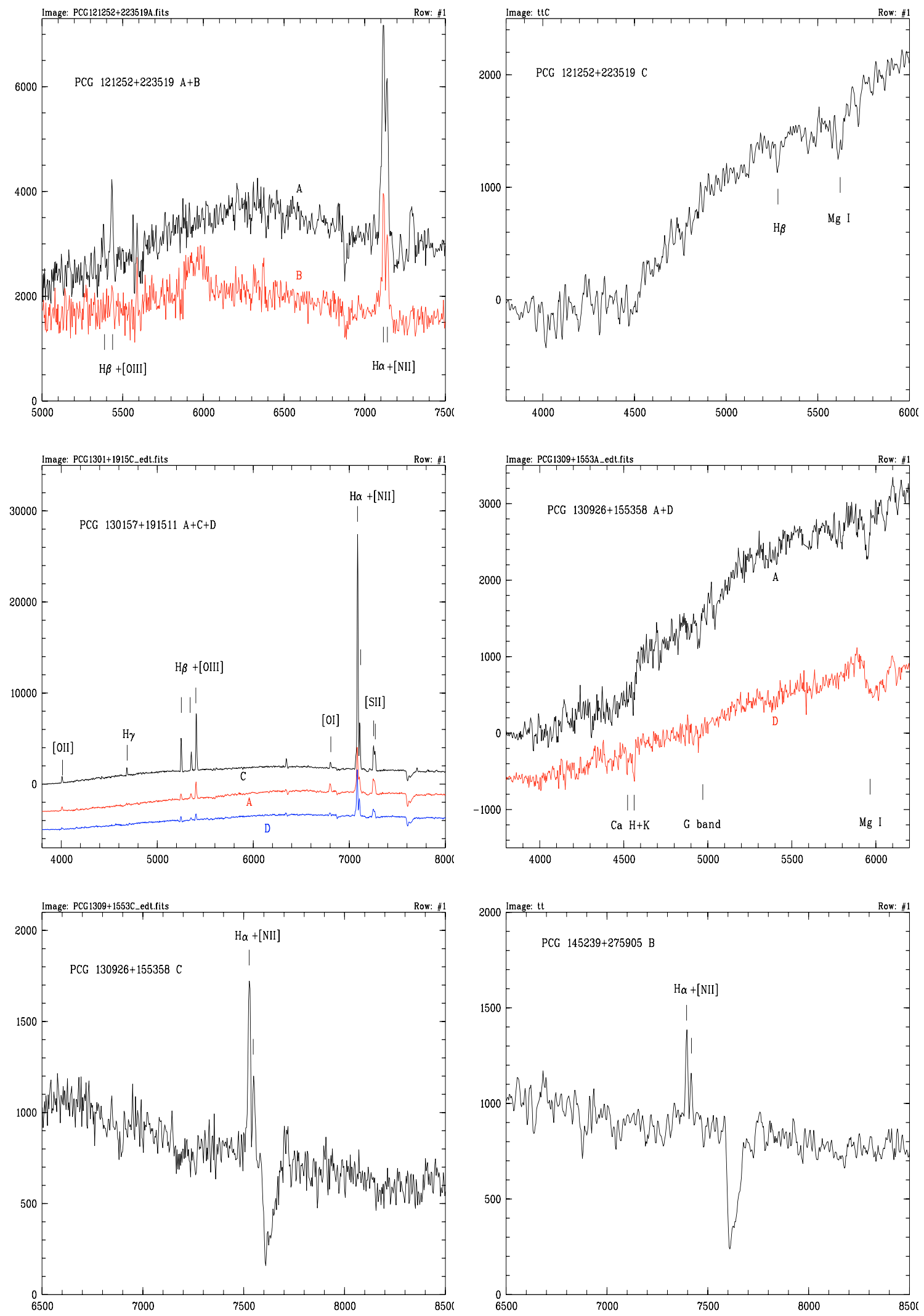

Fig. 3. continued. 
E. Pompei et al.: DPOSS II compact groups survey, Online Material p 9
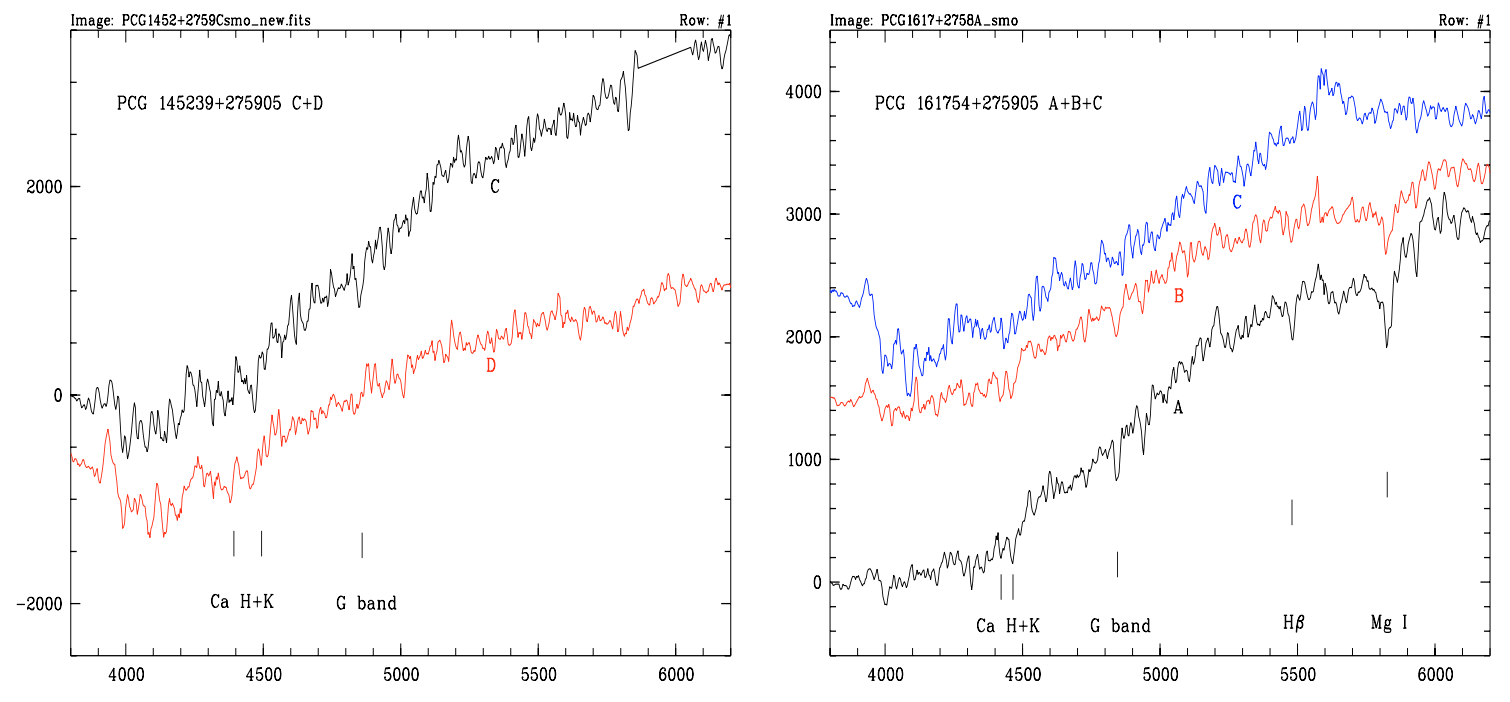

Fig. 3. continued. 